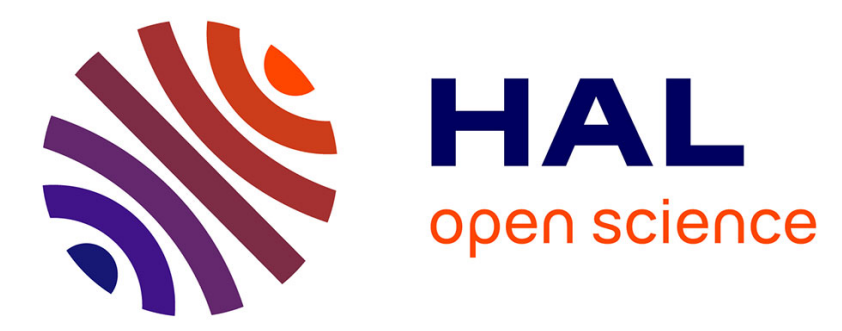

\title{
Combining FFT methods and standard variational principles to compute bounds and estimates for the properties of elastic composites
}

Vincent Monchiet

\section{- To cite this version:}

Vincent Monchiet. Combining FFT methods and standard variational principles to compute bounds and estimates for the properties of elastic composites. Computer Methods in Applied Mechanics and Engineering, 2015, 283, pp.454-473. 10.1016/j.cma.2014.10.005 . hal-01165844

\section{HAL Id: hal-01165844 \\ https://hal.science/hal-01165844}

Submitted on 18 Mar 2021

HAL is a multi-disciplinary open access archive for the deposit and dissemination of scientific research documents, whether they are published or not. The documents may come from teaching and research institutions in France or abroad, or from public or private research centers.
L'archive ouverte pluridisciplinaire HAL, est destinée au dépôt et à la diffusion de documents scientifiques de niveau recherche, publiés ou non, émanant des établissements d'enseignement et de recherche français ou étrangers, des laboratoires publics ou privés. 


\title{
Combining FFT methods and standard variational principles to compute bounds and estimates for the properties of elastic composites
}

\author{
Vincent Monchiet \\ Université Paris-Est, Laboratoire Modélisation et Simulation Multi Echelle, \\ LMSME UMR8208 CNRS, 5 boulevard Descartes, 77454 Marne la Vallée Cedex, \\ France
}

\begin{abstract}
In this paper, we develop a computational approach based on variational principles combined with FFT technique to determine the effective elastic properties of composite materials. The unit cell problem of the composite is recast in a weak and integral form by making use of classic variational approaches, based on the strain and the stress potential, and the Hashin-Shtrikman variational principles. The problem is discretized with Fourier series and the stationary point is computed numerically by means of an iterative scheme. These algorithms use a representation of the local elastic tensor on the double grid (twice the size of the discretization) which is introduced by the integral formulation; it advantageously accounts for the exact geometry of the microstructure and allows the derivation of rigorous bounds of the effective elasticity coefficients. In the second part of the paper, we establish a hierarchy between the different FFT solutions: the strain, the stress based solutions coming from the classic variational formulations and the polarization based solution derived from the Hashin-Shtrikman principle, which depends on the choice of the elastic moduli of the reference material. It is proved that the strain and the stress based solutions deliver optimal bounds since they are always better than those obtained from the Hashin-Shtrikman variational principle when the same discretization is used. Alternatively, when the elastic moduli of the reference material is negative, the polarization based solution provides an estimate of the effective properties which is comprised between the strain based upper bound and the stress based lower bound.
\end{abstract}

Key words: Variational principles, Fast Fourier Transform, composites, homogenization.

Email addresses: vincent.monchiet@univ-paris-est.fr, 


\section{Introduction}

FFT based iterative schemes are simple and powerful methods for solving the unit cell problems and the computation of macroscopic elasticity coefficients of composite materials. The method is based on the resolution of the so-called Lippmann-Schwinger equation by means of a fixed-point method, following an idea already suggested in $[9,15]$. As regards to alternative methods such as finite element, the approach based on FFT present very interesting computational advantages. First, the use of FFT algorithm makes the computation very fast. Next, the memory requirement is strongly reduced compared to Finite Element Method (FEM) or Finite Volume Method (FVM). Instead to need the storage of stiffness or interaction matrices, the storage is required only for the nodal variables and for the components of the Green tensor (6 for $2 \mathrm{D}$ problems and 21 for 3D problems) which are explicitly known in Fourier space.

Since the former numerical algorithm developed by Moulinec et al. [28,29], particular attention has been focused on the convergence of the FFT iterative schemes. Indeed, the rate of convergence of the strain based scheme of Moulinec and Suquet [28], and the dual scheme (based on the stress) of Bhattacharya and Suquet [1], Bonnet [4], depend drastically on the contrast between the elastic coefficients of the constituents. Particularly, the method is not adapted to handle the problem of composites with highly rigid inclusions or voids. This has motivated the formulation of more robust solvers. For instance, accelerated schemes has been proposed by Eyre and Milton [11], Michel et al. [20,21] which has been recently proved in [31] to constitute some particular cases of the polarization based iterative scheme of Monchiet and Bonnet [26]. Note also that the convergence has been also improved by considering the conjugate gradient method to compute iteratively the solution of the Lippman-Schwinger equation $[43,12]$ or by using a modified Green operator in the recurrence relation $[42,41]$.

The FFT methods have been well considered for various applications in the field of linear elastic homogenization $[40,16,10]$ and has also been adapted to other class of linear problems, among which the static and dynamic Darcy problem $[25,33]$ or piezoelectricity $[5,6]$. FFT based iterative schemes have been also successfully applied to non linear constitutive relations including elastoplastic and elastoviscoplastic materials [29,21,30,2,3,35,14,17] and more recently non local damage models $[18,19]$.

The recent developments of the FFT based method have been devoted to their reformulation using variational principles. For instance Brisard and Dormieux $[7,8]$, considered the Hashin-Shtrikman variational principle with constant polarization by pixels for the discretization of the unit cell problem. This has two main consequences on the resulting linear system. First, the real image

tel: (33)0160957793, fax: (33) 0160957795 (Vincent Monchiet). 
of the microstructure is replaced by an array of squared elements as in the former approach $[28,29]$, that only approximates the real geometry for various practical problems such as in the case of fibers inclusions. Next, the method does not use the Green operator, but a periodized Green operator that is not explicitly known and must be evaluated numerically. In another recent contribution [36,37], a link has been established between the Lippmann-Schwinger equation and the Galerkin discretization of the weak form of the unit cell problem.

Variational approaches are interesting in the sense that they provide rigorous bounds for the homogenized elastic coefficients. In this paper we provide a unified variational approach of the basic schemes (based on the strain or the stress) and the polarization schemes. The basic iterative schemes are retrieved using classic variational procedure based on the strain and the stress elastic potential while the polarization scheme is derived in the Hashin-Shtrikman variational approach. The unit cell problem is discretized with Fourier series and the stationarity point are solve by a suitable numerical integration schemes which are formally equivalent to those already proposed in the literature. The method advantageously preserves the explicit form of the Green function in Fourier space but introduces a representation of the local elastic tensor on a double grid, that is twice the dimension of the cell discretization subspace. In the last part of the paper, we provide a comparison of the different FFT solutions: the strain and stress solutions coming from the classic variational procedures and the solution for the polarization. The accuracy of the solution obtained with the different formulations are discussed through numerical examples.

\section{The unit cell problem and Fourier series representation}

Let us consider a composite material represented by a periodic unit cell $V=$ $\prod_{i}\left[-h_{i} / 2, h_{i} / 2\right]$ where $i=1,2$ for $2 \mathrm{~d}$ problems and $i=1,2,3$ for $3 \mathrm{D}$ ones. In the context of elasticity, the unit cell problem reads:

$$
\begin{array}{ll}
\operatorname{div}(\boldsymbol{\sigma})=0 & (\text { equilibrium) } \\
\boldsymbol{\sigma}=\mathbb{C}: \boldsymbol{\varepsilon} & (\text { elastic law) } \\
\boldsymbol{\varepsilon}=\frac{1}{2}\left(\nabla \underline{u}+\nabla^{T} \underline{u}\right) & \text { (compatibility) }
\end{array}
$$

with the following periodicity conditions on the boundary of the unit cell:

$$
\underline{u}-\boldsymbol{E} . \underline{x} \text { periodic, } \boldsymbol{\sigma} . \underline{n} \text { antiperiodic }
$$


In equations (1) and (2), $\underline{u}, \boldsymbol{\varepsilon}$ and $\boldsymbol{\sigma}$ denote the displacement, the strain and the stress fields respectively, $\mathbb{C}$ is the fourth order elasticity tensor and we also denote by $\mathbb{S}=(\mathbb{C})^{-1}$ the compliance.

The problem is enforced with the macroscopic strain $\boldsymbol{E}$,

$$
<\varepsilon>_{V}=\boldsymbol{E}
$$

or the macroscopic stress $\boldsymbol{\Sigma}$,

$$
<\boldsymbol{\sigma}>_{V}=\Sigma
$$

where $\left\langle\bullet>_{V}\right.$ represents the volume average over the unit cell of volume $V$. Since the problem is periodic, it is convenient to work with Fourier series and the associated FFT technique. We consider a representation in Fourier series for the displacement $\underline{u}$, the strain $\varepsilon$, the stress $\boldsymbol{\sigma}$ and other fields considered in the paper such as the polarization $\boldsymbol{\tau}$ and the eigenstrain $\boldsymbol{\eta}$. The expansion in Fourier series of any real quantity $f$ is denoted by:

$$
f(\underline{x})=\sum_{n=-N}^{n=N-1} \widehat{f}\left(\underline{\xi}_{n}\right) \exp \left(i \underline{\xi}_{n} \cdot \underline{x}\right), \quad \widehat{f}(\underline{\xi})=<f(\underline{x}) \exp (-i \underline{\xi} \cdot \underline{x})>_{V}
$$

where $\underline{\xi}_{n}$ denote the discrete wave vectors given by

$$
\underline{\xi}_{n}=2 \pi n \underline{\zeta}, \quad n=-N . . N-1, \quad \zeta_{i}=\frac{1}{h_{i}}
$$

and $h_{1}, h_{2}, h_{3}$ are the half of the cell. Obviously, the problem is discretized along each space direction, this would involve the use of two indices $n_{1}$ and $n_{2}$ for 2-d problems and three for 3-d one. However, only one indice $n$ is used for simplicity. Still for simplicity, we shall use the notation $\widehat{f}_{n}$, the Fourier transform of $f$ associated with the wave vector $\underline{\xi}_{n}$. Particularly, the Fourier component corresponding to $n=0$ of the quantity $f$ represents its average over the volume of the cell, $\widehat{f}_{0}=<f(\underline{x})>_{V}$. Then, the quantity $\widehat{\varepsilon}_{0}=\boldsymbol{E}$ is known when the macroscopic strain $\boldsymbol{E}$ is prescribed to the unit cell. In this case, the macroscopic stress is $\boldsymbol{\Sigma}=\widehat{\boldsymbol{\sigma}}_{0}$ that is determined by the resolution of the unit cell problem. The homogenized elasticity tensor is determined such that $\boldsymbol{\Sigma}=\mathbb{C}^{\text {hom }}: \boldsymbol{E}$ and we also denote by $\mathbb{S}^{\text {hom }}=\left(\mathbb{C}^{\text {hom }}\right)^{-1}$ the homogenized compliance tensor. Alternatively, when the macroscopic stress is applied, the macroscopic strain have to be determined.

In the next section, classic variational principles are considered to obtain elementary bounds for the macroscopic elastic energy. These variational principles are based on the consideration of admissible subsets made up of compatible fields for the strain, and equilibrated fields for the stress. These ad- 
missibility conditions can be formulated by means of two suitable projection operators, $\mathbb{P}_{n}$ and $\mathbb{Q}_{n}$, defined for every $\underline{\xi}_{n} \neq 0$ by:

$$
\mathbb{Q}_{n}=\mathbb{E}_{1}\left(\underline{\xi}_{n}\right)+\mathbb{E}_{3}\left(\underline{\xi}_{n}\right), \quad \mathbb{P}_{n}=\mathbb{E}_{2}\left(\underline{\xi}_{n}\right)+\mathbb{E}_{4}\left(\underline{\xi}_{n}\right)
$$

in which the $\mathbb{E}_{i}(\underline{\xi})$ for $i=1 . .4$ are the first four tensors of the Walpole basis [38]. Let us recall the six tensors of this basis:

$$
\left\{\begin{array}{l}
\mathbb{E}_{1}(\underline{\xi})=\frac{1}{2} \boldsymbol{k}^{\perp} \otimes \boldsymbol{k}^{\perp}, \quad \mathbb{E}_{2}(\underline{\xi})=\boldsymbol{k} \otimes \boldsymbol{k} \\
\mathbb{E}_{3}(\underline{\xi})=\boldsymbol{k}^{\perp} \underline{\bar{\otimes}} \boldsymbol{k}^{\perp}-\mathbb{E}_{1}, \quad \mathbb{E}_{4}(\underline{\xi})=\boldsymbol{k}^{\perp} \underline{\bar{\otimes}} \boldsymbol{k}+\boldsymbol{k} \underline{\otimes} \boldsymbol{k}^{\perp} \\
\mathbb{E}_{5}(\underline{\xi})=\boldsymbol{k} \otimes \boldsymbol{k}^{\perp}, \quad \mathbb{E}_{6}(\underline{\xi})=\boldsymbol{k}^{\perp} \otimes \boldsymbol{k}
\end{array}\right.
$$

where $\boldsymbol{k}$ and $\boldsymbol{k}^{\perp}$ are given by:

$$
\boldsymbol{k}=\frac{1}{|\underline{\xi}|^{2}} \underline{\xi} \otimes \underline{\xi}, \quad \boldsymbol{k}^{\perp}=\boldsymbol{i}-\boldsymbol{k}
$$

and $\boldsymbol{i}$ is the second order identity tensor. As already shown in [26], the strain field is compatible if its fourier coefficients $\widehat{\varepsilon}_{n}$ comply with

$$
\forall n \neq 0, \quad \mathbb{Q}_{n}: \widehat{\varepsilon}_{n}=0
$$

Alternatively, the equilibrium for the stress field leads to the following condition for its fourier coefficients $\widehat{\boldsymbol{\sigma}}_{n}$ :

$$
\forall n \neq 0, \quad \mathbb{P}_{n}: \widehat{\boldsymbol{\sigma}}_{n}=0
$$

With these notations, the subset of kinematical and statical admissible fields, denoted $\mathcal{E}_{N}$ and $\mathcal{S}_{N}$ respectively, are defined by:

$$
\begin{aligned}
& \mathcal{E}_{N}=\left\{\boldsymbol{\varepsilon}=\sum_{n=-N}^{n=N-1} \widehat{\boldsymbol{\varepsilon}}_{n} \exp \left(i \underline{\xi}_{n} \cdot \underline{x}\right), \widehat{\boldsymbol{\varepsilon}}_{0}=\boldsymbol{E}, \mathbb{Q}_{n}: \widehat{\boldsymbol{\varepsilon}}_{n}=0 \text { for } n \neq 0\right\} \\
& \mathcal{S}_{N}=\left\{\boldsymbol{\sigma}=\sum_{n=-N}^{n=N-1} \widehat{\boldsymbol{\sigma}}_{n} \exp \left(i \underline{\xi}_{n} \cdot \underline{x}\right), \widehat{\boldsymbol{\sigma}}_{0}=\boldsymbol{\Sigma}, \mathbb{P}_{n}: \widehat{\boldsymbol{\sigma}}_{n}=0 \text { for } n \neq 0\right\}
\end{aligned}
$$

In the next section, we derive the solutions $\varepsilon \in \mathcal{E}_{N}$ and $\boldsymbol{\sigma} \in \mathcal{S}_{N}$ within the classic variational principles. 


\section{Classic variational principles with FFT numerical integration}

Variational principles based on the macroscopic elastic energy are now considered to determine a lower and an upper bound for the effective elastic coefficients of the composites. Let us introduce the strain elastic energy $\mathcal{U}(\varepsilon)$ and the complementary elastic potential $\mathcal{W}(\boldsymbol{\sigma})$ by:

$$
\mathcal{U}(\varepsilon)=\frac{1}{2}<\varepsilon: \mathbb{C}: \varepsilon>_{V}, \quad \mathcal{W}(\boldsymbol{\sigma})=\frac{1}{2}<\boldsymbol{\sigma}: \mathbb{S}: \boldsymbol{\sigma}>_{V}
$$

Since the elastic tensors $\mathbb{C}$ and $\mathbb{S}$ are positive definite, the following energy minimization principles hold for the macroscopic elastic energy (see for instance [34]):

$$
\begin{aligned}
& \frac{1}{2} \boldsymbol{E}: \mathbb{C}^{\text {hom }}: \boldsymbol{E} \leq \frac{1}{2} \boldsymbol{E}: \mathbb{C}_{N}^{\varepsilon}: \boldsymbol{E}=\min _{\boldsymbol{\varepsilon} \in \mathcal{E}_{N}} \mathcal{U}(\boldsymbol{\varepsilon}) \\
& \frac{1}{2} \boldsymbol{\Sigma}: \mathbb{S}^{h o m}: \boldsymbol{\Sigma} \leq \frac{1}{2} \boldsymbol{\Sigma}: \mathbb{S}_{N}^{\sigma}: \boldsymbol{\Sigma}=\min _{\boldsymbol{\sigma} \in \mathcal{S}_{N}} \mathcal{W}(\boldsymbol{\sigma})
\end{aligned}
$$

where $\mathbb{C}_{N}^{\varepsilon}$ and $\mathbb{S}_{N}^{\sigma}$ are two estimates of the homogenized elastic tensor $\mathbb{C}^{\text {hom }}=$ $\left(\mathbb{S}^{h o m}\right)^{-1}$ in which the index $N$ refers to the dimension of the subset of admissible fields for the strain and the stress. Classically, the following inequality is deduced for the elasticity tensors:

$$
\mathbb{C}_{N}^{\sigma} \leq\left(\mathbb{S}^{h o m}\right)^{-1}=\mathbb{C}^{h o m} \leq \mathbb{C}_{N}^{\varepsilon}
$$

with the notation $\mathbb{C}_{N}^{\sigma}=\left(\mathbb{S}_{N}^{\sigma}\right)^{-1}$.

Replacing, in the expressions (12), the strain and stress fields by their Fourier series and taking advantage of Parseval's theorem, one obtains:

$$
\begin{aligned}
& \mathcal{U}(\varepsilon)=\tilde{\mathcal{U}}\left(\widehat{\boldsymbol{\varepsilon}}_{n}\right)=\frac{1}{2} \sum_{n=-N}^{n=N-1} \sum_{m=-N}^{m=N-1} \overline{\widehat{\boldsymbol{\varepsilon}}_{n}}: \widehat{\mathbb{C}}_{n-m}: \widehat{\boldsymbol{\varepsilon}}_{m}, \\
& \mathcal{W}(\boldsymbol{\sigma})=\widetilde{\mathcal{W}}\left(\widehat{\boldsymbol{\sigma}}_{n}\right)=\frac{1}{2} \sum_{n=-N}^{n=N-1} \sum_{m=-N}^{m=N-1} \widehat{\boldsymbol{\sigma}}_{n}: \widehat{\mathbb{S}}_{n-m}: \widehat{\boldsymbol{\sigma}}_{m}
\end{aligned}
$$

Where the quantity $\overline{\widehat{\boldsymbol{a}}_{n}}$ denotes the conjugate of $\widehat{\boldsymbol{a}}_{n}$. Moreover, $\widehat{\mathbb{C}}_{n}$ and $\widehat{\mathbb{S}}_{n}$ are the Fourier coefficients of the elasticity tensor. For a composite made up of $p$ distinct elastic constituents, the elasticity tensors can be decomposed in the form: 


$$
\mathbb{C}=\sum_{\alpha=1}^{\alpha=p} \mathbb{C}^{\alpha} I^{\alpha}(\underline{x}), \quad \mathbb{S}=\sum_{\alpha=1}^{\alpha=p} \mathbb{S}^{\alpha} I^{\alpha}(\underline{x})
$$

where $I^{\alpha}(\underline{x})$ are the characteristic functions of the phases, defined by:

$$
I^{\alpha}(\underline{x})=\left\{\begin{array}{l}
1 \text { if } \underline{x} \in V_{\alpha} \\
0 \text { if } \underline{x} \in V-V_{\alpha}
\end{array}\right.
$$

where $\mathbb{C}^{\alpha}$ and $\mathbb{S}^{\alpha}=\left(\mathbb{C}^{\alpha}\right)^{-1}$ are the constant elasticity tensors of the individual constituents. The discrete convolution product between a quantity $\widehat{y}_{n}$ and the Fourier coefficients of a characteristic function is denoted

$$
\widehat{y}_{n}^{\alpha}=\sum_{m=-N}^{m=N-1} \widehat{I}_{n-m}^{\alpha} \widehat{y}_{m}
$$

With these notations, the expressions in (15) also read:

$$
\begin{aligned}
& \widetilde{\mathcal{U}}\left(\widehat{\boldsymbol{\sigma}}_{n}\right)=\frac{1}{2} \sum_{n=-N}^{n=N-1} \overline{\widehat{\boldsymbol{\varepsilon}}_{n}}:\left[\sum_{\alpha=1}^{\alpha=p} \mathbb{C}^{\alpha}: \widehat{\boldsymbol{\varepsilon}}_{n}^{\alpha}\right], \\
& \widetilde{\mathcal{W}}\left(\widehat{\boldsymbol{\sigma}}_{n}\right)=\frac{1}{2} \sum_{n=-N}^{n=N-1} \overline{\widehat{\boldsymbol{\sigma}}_{n}}:\left[\sum_{\alpha=1}^{\alpha=p} \mathbb{S}^{\alpha}: \widehat{\boldsymbol{\sigma}}_{n}^{\alpha}\right]
\end{aligned}
$$

The stationarity of $\tilde{\mathcal{U}}\left(\widehat{\varepsilon}_{n}\right)$ with respect to $\widehat{\varepsilon}_{n}$ (accounting for the admissibility conditions (11)) leads to the following linear system for the minimizer $\widehat{\varepsilon}_{n}$ :

$$
\left\{\begin{array}{l}
\mathbb{Q}_{n}: \widehat{\boldsymbol{\varepsilon}}_{n}=0 \quad n \neq 0, \\
\mathbb{P}_{n}:\left[\sum_{\alpha=1}^{\alpha=p} \mathbb{C}^{\alpha}: \widehat{\boldsymbol{\varepsilon}}_{n}^{\alpha}\right]=0 \quad n \neq 0 \\
\widehat{\boldsymbol{\varepsilon}}_{0}=\boldsymbol{E}
\end{array}\right.
$$

Conversely, the stationarity of $\widetilde{\mathcal{W}}\left(\widehat{\boldsymbol{\sigma}}_{n}\right)$ with respect to $\widehat{\boldsymbol{\sigma}}_{n}$ (accounting for the admissibility conditions (11)) gives: 


$$
\left\{\begin{array}{l}
\mathbb{P}_{n}: \widehat{\boldsymbol{\sigma}}_{n}=0 \quad n \neq 0 \\
\mathbb{Q}_{n}:\left[\sum_{\alpha=1}^{\alpha=p} \mathbb{S}^{\alpha}: \widehat{\boldsymbol{\sigma}}_{n}^{\alpha}\right]=0 \quad n \neq 0 \\
\widehat{\boldsymbol{\sigma}}_{0}=\mathbf{\Sigma}
\end{array}\right.
$$

The direct resolution of both linear systems (20) and (21) is computationally too expensive due to the presence of the discrete convolution product between the fourier coefficients of the characteristic functions of the phase and those of the strain and stress fields. The resolution by means of an iterative scheme which uses the FFT algorithm is obviously recommended to reduce drastically the computer memory requirements and the CPU time. For instance, strain based iterative schemes introduced by $[28,29]$ and the stress formulations $[1,4]$ can be used to compute the solution of the linear systems (20) and (21). The solution of (20) is computed with the following recurrence relation:

$$
\left(\widehat{\boldsymbol{\varepsilon}}_{n}\right)^{i+1}=\left(\widehat{\boldsymbol{\varepsilon}}_{n}\right)^{i}-\widehat{\boldsymbol{\Gamma}}_{n}^{0}:\left[\sum_{\alpha=1}^{\alpha=p} \mathbb{C}^{\alpha}:\left(\widehat{\boldsymbol{\varepsilon}}_{n}^{\alpha}\right)^{i}\right]
$$

which starts with $\widehat{\boldsymbol{\varepsilon}}_{n}=0$ for $n \neq 0$ and $\widehat{\boldsymbol{\varepsilon}}_{0}=\boldsymbol{E}$. In $(22), \widehat{\boldsymbol{\Gamma}}_{n}^{0}$ are the Fourier coefficients of the Green tensor associated to the reference material of rigidity $\mathbb{C}^{0}$. The iterative scheme $(22)$ can be interpreted as a fixed-point method for computing the solution of the linear system (20). A priori, tensor $\mathbb{P}_{n}$ could be used instead of the Green operator, however the latter is required to reach the convergence. Indeed, $\Gamma^{0}$ depends on the elastic coefficients of the reference material which are chosen such that the spectral radius of the operator involved in the iterative scheme is lower than 1 . The analysis of convergence is not the central issue of this work which, besides, has been well documented in the literature. The reader could refer to $[22,24,26,31]$ for more details on these issues. The introduction of the Green operator could be interpreted as preconditioning since there is close relationship between tensor $\mathbb{P}_{n}$ and the Green tensor. Indeed, for an isotropic elastic reference material, the Green operator can be decomposed along the Walpole basis:

$$
\forall n \neq 0: \widehat{\boldsymbol{\Gamma}}_{n}^{0}=\frac{1}{\lambda_{0}+2 \mu_{0}} \mathbb{E}_{2}\left(\underline{\xi}_{n}\right)+\frac{1}{2 \mu_{0}} \mathbb{E}_{4}\left(\underline{\xi}_{n}\right)
$$

and $\widehat{\boldsymbol{\Gamma}}_{n}^{0}=0$ for $n=0$. In (23), $\lambda_{0}$ and $\mu_{0}$ are the Lamé coefficients of the reference medium. Tensor $\mathbb{P}_{n}$ and $\widehat{\boldsymbol{\Gamma}}_{n}^{0}$ are then represented with the same tensors of the Walpole basis. It follows that the first equation in (20) remains unchanged if $\mathbb{P}_{n}$ is replaced by $\boldsymbol{\Gamma}_{n}^{0}$. Moreover, tensor $\mathbb{P}_{n}$ is equal to $\widehat{\boldsymbol{\Gamma}}_{n}^{0}$ when $\lambda_{0}=0$ and $\mu_{0}=1 / 2$. Note that the Green tensor is transversely isotropic with respect to the direction given by the wave vector $\underline{\xi}$ and can be decomposed along the 
Walpole basis only if the reference material is isotropic. Such decomposition is obviously not true for other class of material symmetries.

Similarly, the solution of the linear system (21) can be computed by means of a stress based iterative scheme:

$$
\left(\widehat{\boldsymbol{\sigma}}_{n}\right)^{i+1}=\left(\widehat{\boldsymbol{\sigma}}_{n}\right)^{i}-\widehat{\boldsymbol{\Delta}}_{n}^{0}:\left[\sum_{\alpha=1}^{\alpha=p} \mathbb{S}^{\alpha}:\left(\widehat{\boldsymbol{\sigma}}_{n}^{\alpha}\right)^{i}\right]
$$

which is initialized with $\widehat{\boldsymbol{\sigma}}_{n}=0$ for any $n \neq 0$ and $\widehat{\boldsymbol{\sigma}}_{0}=\boldsymbol{\Sigma}$. In $(24), \widehat{\boldsymbol{\Delta}}_{n}^{0}$ are the Fourier coefficients of the dual Green tensor defined by:

$$
\widehat{\boldsymbol{\Delta}}_{n}^{0}=\mathbb{C}^{0}-\mathbb{C}^{0}: \widehat{\boldsymbol{\Gamma}}_{n}^{0}: \mathbb{C}^{0}=\frac{2 \mu_{0}\left(3 \lambda_{0}+2 \mu_{0}\right)}{\lambda_{0}+2 \mu_{0}} \mathbb{E}_{1}\left(\underline{\xi}_{n}\right)+2 \mu_{0} \mathbb{E}_{3}\left(\underline{\xi}_{n}\right)
$$

for $n \neq 0$ and $\widehat{\boldsymbol{\Delta}}_{n}^{0}=0$ for $n=0$.

Again the introduction of $\widehat{\boldsymbol{\Delta}}_{n}^{0}$ in (24) can be interpreted as preconditioning. The convergence test used for the strain and the stress based iterative schemes are based on the residual of the linear system (20) and (20):

$$
\begin{aligned}
& \text { strain scheme: }\left\|\mathbb{P}_{n}:\left[\sum_{\alpha=1}^{\alpha=p} \mathbb{C}^{\alpha}:\left(\widehat{\boldsymbol{\varepsilon}}_{n}^{\alpha}\right)^{i}\right]\right\|<\epsilon \\
& \text { stress scheme: }\left\|\mathbb{Q}_{n}:\left[\sum_{\alpha=1}^{\alpha=p} \mathbb{S}^{\alpha}:\left(\widehat{\boldsymbol{\sigma}}_{n}^{\alpha}\right)^{i}\right]\right\|<\epsilon
\end{aligned}
$$

where the precision $\epsilon=10^{-4}$ is considered in the applications.

It is worth noted that the iterative schemes (22) and (24) are formally equivalent to that introduced in $[28,29]$ and $[1,4]$. They use the Green operator that is explicitly determined by the wave vectors when it is represented in the Fourier space. Its components only requires the storage of tables of dimension $(2 N)^{d}$ for d-dimensional problems. However the difference comes from the presence of the discrete convolution product with the Fourier coefficients of the characteristic functions of the phase, $\hat{I}_{n}^{\alpha}$, which is performed on the double grid. The details are provided in section 5 .

\section{Computation of cell problem with Hashin-Shtrikman variational principles}

We shall use now the Hashin-Shtrikman variational principle to compute the solution of the unit cell problem. There are basically four Hashin-Shtrikman inequalities: two upper bounds and two lower bounds derived from a primal 
and dual variational principle which are recalled in sections 4.1 and 4.2. The equivalence between dicretized solution of the primal and the dual variational principles is established in section 4.2 .

\subsection{Primal Principle}

The primal variational formulation of Hashin-Shtrikman [13,39] involves the introduction of a reference material $\mathbb{C}^{0}$ and the functional $\mathcal{H}(\boldsymbol{\tau})$ defined by

$$
\mathcal{H}(\boldsymbol{\tau})=\frac{1}{2} \boldsymbol{E}: \mathbb{C}^{0}: \boldsymbol{E}+<\boldsymbol{\tau}>_{V}: \boldsymbol{E}-\frac{1}{2}<\boldsymbol{\tau}: \delta \mathbb{C}^{-1}: \boldsymbol{\tau}+\boldsymbol{\tau}: \boldsymbol{\Gamma}^{0} * \boldsymbol{\tau}>(27)
$$

which is function of the polarization $\boldsymbol{\tau}$. In $(27), \delta \mathbb{C}^{-1}=\left(\mathbb{C}-\mathbb{C}^{0}\right)^{-1}$ while the symbol "*" denotes the convolution product defined by:

$$
\Gamma^{0} * \boldsymbol{\tau}=\frac{1}{V} \int_{V} \Gamma^{0}\left(\underline{x}-\underline{x}^{\prime}\right): \boldsymbol{\tau}\left(\underline{x}^{\prime}\right) d \underline{x}^{\prime}
$$

The stationarity of (27) leads to a lower or an upper bound for the macroscopic elastic energy, depending on whether $\delta \mathbb{C}^{-1}+\boldsymbol{\Gamma}^{0}$ is either negative or positive definite. Let us recall some classic results (see for instance [24]):

- if $0<\mathbb{C}^{0}<\mathbb{C}$, the operator $\delta \mathbb{C}^{-1}+\Gamma^{0}$ is negative definite, and the HashinShtrikman variational principle leads to a lower bound for the elastic strain energy.

- Conversely, if $\mathbb{C}_{0}>\mathbb{C}$, the operator $\delta \mathbb{C}^{-1}+\Gamma^{0}$ is positive definite, and the Hashin-Shtrikman variational principle leads to an upper bound for the elastic strain energy.

- The situation corresponding to $\mathbb{C}^{0}<0$, has not been yet explored. However, if none of the aforementioned hypotheses is made for $\mathbb{C}^{0}$, the extremal point provides on estimate for the elastic energy that is not necessarily an upper or a lower bound.

The following inequalities hold for the macroscopic elastic energy:

$$
\max _{\substack{\boldsymbol{\tau} \\ 0<\mathbb{C}^{0}<\mathbb{C}}} \mathcal{H}(\boldsymbol{\tau}) \leq \frac{1}{2} \boldsymbol{E}: \mathbb{C}^{\text {hom }}: \boldsymbol{E} \leq \min _{\substack{\boldsymbol{\tau} \\ \mathbb{C}_{0}>\mathbb{C}}} \mathcal{H}(\boldsymbol{\tau})
$$

By adopting a Fourier series representation for the polarization $\boldsymbol{\tau}$, the HashinShtrikman functional (27) takes the form: 


$$
\begin{aligned}
\mathcal{H}(\boldsymbol{\tau})= & \frac{1}{2} \boldsymbol{E}: \mathbb{C}^{0}: \boldsymbol{E}+\widehat{\boldsymbol{\tau}}_{0}: \boldsymbol{E}-\frac{1}{2} \sum_{n=-N}^{n=N-1} \overline{\widehat{\boldsymbol{\tau}}}_{n}: \widehat{\boldsymbol{\Gamma}}_{n}^{0}: \widehat{\boldsymbol{\tau}}_{n} \\
& -\frac{1}{2} \sum_{n=-N}^{n=N-1} \overline{\widehat{\boldsymbol{\tau}}_{n}}:\left[\sum_{\alpha=1}^{\alpha=p}\left(\delta \mathbb{C}^{\alpha}\right)^{-1}: \widehat{\boldsymbol{\tau}}_{n}^{\alpha}\right]
\end{aligned}
$$

The stationary of $\mathcal{H}(\boldsymbol{\tau})$ is reached for:

$$
\sum_{\alpha=1}^{\alpha=p}\left(\delta \mathbb{C}^{\alpha}\right)^{-1}: \widehat{\boldsymbol{\tau}}_{n}^{\alpha}+\widehat{\boldsymbol{\Gamma}}_{n}^{0}: \widehat{\boldsymbol{\tau}}_{n}=\delta_{n} \boldsymbol{E}
$$

for $n=-N . . N-1$ and where $\delta_{n}=0$ for any $n \neq 0$ but takes the value 1 for $n=0$.

This linear system can be put into an alternative form, more convenient for its numerical resolution. Considering the following properties of the Green tensor:

$$
\forall n \neq 0: \quad \mathbb{Q}_{n}: \widehat{\boldsymbol{\Gamma}}_{n}^{0}=0, \quad \mathbb{P}_{n}: \mathbb{C}^{0}: \widehat{\boldsymbol{\Gamma}}_{n}^{0}=\mathbb{P}_{n}
$$

in relation (31), leads to:

$$
\begin{aligned}
& \mathbb{Q}_{n}:\left[\sum_{\alpha=1}^{\alpha=p}\left(\delta \mathbb{C}^{\alpha}\right)^{-1}: \widehat{\boldsymbol{\tau}}_{n}^{\alpha}\right]=0 \\
& \mathbb{P}_{n}:\left[\sum_{\alpha=1}^{\alpha=p} \mathbb{C}^{\alpha}:\left(\delta \mathbb{C}^{\alpha}\right)^{-1}: \widehat{\boldsymbol{\tau}}_{n}^{\alpha}\right]=0
\end{aligned}
$$

Additionally, by putting $n=0$ in (31), we obtain:

$$
\sum_{\alpha=1}^{\alpha=p}\left(\delta \mathbb{C}^{\alpha}\right)^{-1}: \widehat{\boldsymbol{\tau}}_{0}^{\alpha}-\boldsymbol{E}=0
$$

To summarize the solution of (31) is equivalently solution of the linear system constituted of (33) and (34). The solution can be computed with the following polarization-based iterative scheme:

$$
\begin{aligned}
&\left(\widehat{\boldsymbol{\tau}}_{n}\right)^{i+1}=\left(\widehat{\boldsymbol{\tau}}_{n}\right)^{i}-\alpha \mathbb{C}^{0}: \widehat{\boldsymbol{\Gamma}}_{n}^{0}:\left[\sum_{\alpha=1}^{\alpha=p} \mathbb{C}^{\alpha}:\left(\delta \mathbb{C}^{\alpha}\right)^{-1}:\left(\widehat{\boldsymbol{\tau}}_{n}^{\alpha}\right)^{i}\right] \\
&-\beta \widehat{\boldsymbol{\Delta}}_{n}^{0}:\left[\sum_{\alpha=1}^{\alpha=p}\left(\delta \mathbb{C}^{\alpha}\right)^{-1}:\left(\widehat{\boldsymbol{\tau}}_{n}^{\alpha}\right)^{i}\right], \text { for } n \neq 0 \\
&\left(\widehat{\boldsymbol{\tau}}_{0}\right)^{i+1}=\left(\widehat{\boldsymbol{\tau}}_{0}\right)^{i}-\gamma \mathbb{C}^{0}:\left[\sum_{\alpha=1}^{\alpha=p}\left(\delta \mathbb{C}^{\alpha}\right)^{-1}:\left(\widehat{\boldsymbol{\tau}}_{0}^{\alpha}\right)^{i}-\boldsymbol{E}\right]
\end{aligned}
$$


and which starts from:

$$
\widehat{\tau}_{n}^{i=1}=0, \text { for } n=-N . . N-1
$$

In (35), $\alpha, \beta$, and $\gamma$ are three coefficients introduced to obtain the convergence of the polarization based iterative scheme. Considering an isotropic reference material, and denoting by $k_{0}$ and $\mu_{0}$ its compressibility and the shear modulus, the convergence is ensured if $[26,27]$ :

$$
\begin{array}{ll}
0<\frac{\alpha k}{k-k_{0}}<2, & 0<\frac{\alpha \mu}{\mu-\mu_{0}}<2 \\
0<\frac{\beta k_{0}}{k-k_{0}}<2, & 0<\frac{\beta \mu_{0}}{\mu-\mu_{0}}<2 \\
0<\frac{\gamma k_{0}}{k-k_{0}}<2, & 0<\frac{\gamma \mu_{0}}{\mu-\mu_{0}}<2
\end{array}
$$

Since the conditions involving $\gamma$ and $\beta$ are the same, we put $\gamma=\beta$. Depending on the values of $k_{0}$ and $\mu_{0}$ the following conditions for the convergence are derived from (37):

- if $0<k_{0}<k_{\min }$ and $0<\mu_{0}<\mu_{m i n}$, the convergence is satisfied for:

$$
\begin{array}{ll}
0<\alpha<2\left(1-\frac{k_{0}}{k_{\text {min }}}\right), & 0<\beta<2\left(\frac{k_{\text {min }}}{k_{0}}-1\right) \\
0<\alpha<2\left(1-\frac{\mu_{0}}{\mu_{\text {min }}}\right), & 0<\beta<2\left(\frac{\mu_{\text {min }}}{\mu_{0}}-1\right)
\end{array}
$$

- Conversely, if $k_{0}>k_{\max }$ and $\mu_{0}>\mu_{\max }$ the values of $\alpha$ and $\beta$ must be chosen such that:

$$
\begin{aligned}
& 2\left(1-\frac{k_{0}}{k_{\max }}\right)<\alpha<0, \quad 2\left(\frac{k_{\max }}{k_{0}}-1\right)<\beta<0 \\
& 2\left(1-\frac{\mu_{0}}{\mu_{\max }}\right)<\alpha<0, \quad 2\left(\frac{\mu_{\max }}{\mu_{0}}-1\right)<\beta<0
\end{aligned}
$$

- If $k_{\min }<k_{0}<k_{\max }$ and $\mu_{\min }<\mu_{0}<\mu_{\max }$, there no values for $\alpha$ and $\beta$ which satisfy (37). This case must be excluded when using the polarization based iterative scheme.

- if $k_{0}<0$ and $\mu_{0}<0$, the convergence is reached for:

$$
\begin{array}{ll}
0<\alpha<2\left(1-\frac{k_{0}}{k_{\text {min }}}\right), & 2\left(\frac{k_{\text {min }}}{k_{0}}-1\right)<\beta<0 \\
0<\alpha<2\left(1-\frac{\mu_{0}}{\mu_{\text {min }}}\right), & 2\left(\frac{\mu_{\text {min }}}{\mu_{0}}-1\right)<\beta<0
\end{array}
$$


Particularly, when $0<\alpha<2$ and $-2<\beta<0$, the convergence is reached independently of the local elasticity coefficients since $k_{0} / k_{\text {min }}$ and $\mu_{0} / \mu_{\text {min }}$ are negative quantities.

Conditions (38) and (39) are considered for computing the lower bound and the upper bound respectively. Conditions (40) can be also used, but the corresponding values for the homogenized elastic properties is uncertain and will be further investigated.

From a practical point of view and for the applications proposed in the sequel, the following values of $\alpha$ and $\beta$ are considered depending on whether we aim at computing the lower bound, the upper bound or an intermediate solution (the terminology "intermediate" refers to the solution of the linear system (31) computed with negative elastic moduli for the reference material):

- Lower bound:

$\alpha=\min \left(1-\frac{k_{0}}{k_{\min }}, 1-\frac{\mu_{0}}{\mu_{\min }}\right), \quad \beta=\min \left(\frac{k_{\min }}{k_{0}}-1, \frac{\mu_{\min }}{k_{0}}-1\right)$

- Upper bound:

$\alpha=\max \left(1-\frac{k_{0}}{k_{\max }}, 1-\frac{\mu_{0}}{\mu_{\max }}\right), \quad \beta=\max \left(\frac{k_{\max }}{k_{0}}-1, \frac{\mu_{\max }}{k_{0}}-1\right)$

- Intermediate: $\alpha=1, \quad \beta=-1$

These choices are quite optimal as regards to the numerical results.

A convergence test based on the residual of the linear system (33) together with (34) is used:

$$
\begin{aligned}
& \left\|\mathbb{P}_{n}:\left[\sum_{\alpha=1}^{\alpha=p} \mathbb{C}^{\alpha}:\left(\delta \mathbb{C}^{\alpha}\right)^{-1}:\left(\widehat{\boldsymbol{\tau}}_{n}^{\alpha}\right)^{i}\right]\right\|<\epsilon, \\
& \left\|\mathbb{Q}_{n}:\left[\sum_{\alpha=1}^{\alpha=p}\left(\delta \mathbb{C}^{\alpha}\right)^{-1}:\left(\widehat{\boldsymbol{\tau}}_{n}^{\alpha}\right)^{i}\right]\right\|<\epsilon, \\
& \left\|\sum_{\alpha=1}^{\alpha=p}\left(\delta \mathbb{C}^{\alpha}\right)^{-1}:\left(\hat{\boldsymbol{\tau}}_{0}^{\alpha}\right)^{i}-\boldsymbol{E}\right\|<\epsilon
\end{aligned}
$$

At stationarity, $\mathcal{H}(\boldsymbol{\tau})$ reads:

$$
\mathcal{H}(\boldsymbol{\tau})=\frac{1}{2}\left(\widehat{\boldsymbol{\tau}}_{0}+\mathbb{C}^{0}: \boldsymbol{E}\right): \boldsymbol{E}
$$

The solution of (31) linearly depends on the macroscopic strain $\boldsymbol{E}$. Particularly, for $n=0$, we can introduce the fourth order tensor $\mathbb{C}_{N}^{\tau}$ such that $\widehat{\boldsymbol{\tau}}_{0}=\left(\mathbb{C}_{N}^{\tau}-\mathbb{C}^{0}\right): \boldsymbol{E}$. With this notation, the value of $\mathcal{H}(\boldsymbol{\tau})$ at the extremal point is: 


$$
\mathcal{H}(\boldsymbol{\tau})=\frac{1}{2} \boldsymbol{E}: \mathbb{C}_{N}^{\tau}: \boldsymbol{E}
$$

However the solution of the linear system defined by equations (33) and (34) depends on the choice of the reference material, $\mathbb{C}_{N}^{\tau}$ is then a function of $\mathbb{C}^{0}$. From (29) we deduce the following inequalities:

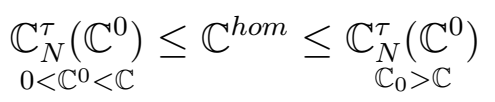

Interestingly, it must be noted that the linear systems (20) and (21) for the strain and stress are two particular cases of (33) together with (34) when taking the limits $\mathbb{C}^{0} \rightarrow 0$ and $\mathbb{C}^{0} \rightarrow \pm \infty$. Indeed, when $\mathbb{C}^{0} \rightarrow 0$, then $\left(\delta \mathbb{C}^{\alpha}\right)^{-1}=\mathbb{S}^{\alpha}$ and the linear system $(21)$ for the stress is recovered. Alternatively, if we replace $\boldsymbol{\tau}$ by $\mathbb{C}^{0}: \varepsilon$ and by taking the limit $\mathbb{C}^{0} \rightarrow \pm \infty$ in relations (33) and (34), the linear system (20) for the strain is recovered. This suggests that:

$$
\begin{aligned}
& \lim _{\mathbb{C}^{0} \rightarrow \pm \infty} \mathbb{C}_{N}^{\tau}\left(\mathbb{C}^{0}\right)=\mathbb{C}_{N}^{\varepsilon} \\
& \lim _{\mathbb{C}^{0} \rightarrow 0} \mathbb{C}_{N}^{\tau}\left(\mathbb{C}^{0}\right)=\mathbb{C}_{N}^{\sigma}
\end{aligned}
$$

\subsection{Dual Principle}

The effective elasticity coefficients of the composites can be computed by the dual formulation of the Hashin-Shtrikman variational principle [23,44]. This dual form uses the eigenstrain $\boldsymbol{\eta}$, the dual Green operator $\boldsymbol{\Delta}^{0}$ and the compliance $\mathbb{S}$ and deals with the unit cell problem with an applied macroscopic stress. The following complementary inequalities hold for the macroscopic elastic energy:

$$
\max _{\substack{\eta \\ 0<\mathbb{S}^{0}<\mathbb{S}}} \mathcal{L}(\boldsymbol{\eta}) \leq \frac{1}{2} \boldsymbol{\Sigma}: \mathbb{S}^{\text {hom }}: \boldsymbol{\Sigma} \leq \min _{\substack{\boldsymbol{\eta} \\ \mathbb{S}^{0}>\mathbb{S}}} \mathcal{L}(\boldsymbol{\eta})
$$

where $\mathcal{L}(\boldsymbol{\eta})$ is defined by:

$$
\mathcal{L}(\boldsymbol{\eta})=\frac{1}{2} \boldsymbol{\Sigma}: \mathbb{S}^{0}: \boldsymbol{\Sigma}+<\boldsymbol{\eta}>_{V}: \boldsymbol{\Sigma}-\frac{1}{2}<\boldsymbol{\eta}: \delta \mathbb{S}^{-1}: \boldsymbol{\eta}+\boldsymbol{\eta}: \boldsymbol{\Delta}^{0} * \boldsymbol{\eta}>_{V}
$$

and $\delta \mathbb{S}=\mathbb{S}-\mathbb{S}^{0}$. The linear system associated with the dual variational formulation is: 


$$
\sum_{\alpha=1}^{\alpha=p}\left(\delta \mathbb{S}^{\alpha}\right)^{-1}: \widehat{\boldsymbol{\eta}}_{n}^{\alpha}+\widehat{\boldsymbol{\Delta}}_{n}^{0}: \widehat{\boldsymbol{\eta}}_{n}=\delta_{n} \boldsymbol{\Sigma}
$$

for $n=-N . . N-1$.

At the stationarity point, the value of $\mathcal{L}(\boldsymbol{\eta})$ is:

$$
\mathcal{L}(\boldsymbol{\eta})=\frac{1}{2}\left(\widehat{\boldsymbol{\eta}}_{0}+\mathbb{S}^{0}: \boldsymbol{\Sigma}\right): \boldsymbol{\Sigma}
$$

Let us introduce the fourth order tensor $\mathbb{S}_{N}^{\eta}$ such that $\widehat{\boldsymbol{\eta}}_{0}=\left(\mathbb{S}_{N}^{\eta}-\mathbb{S}^{0}\right): \boldsymbol{\Sigma}$. The value of $\mathcal{L}(\boldsymbol{\eta})$ at the extremal point is:

$$
\mathcal{L}(\boldsymbol{\eta})=\frac{1}{2} \boldsymbol{\Sigma}: \mathbb{S}_{N}^{\eta}: \boldsymbol{\Sigma}
$$

However, the following equivalence hold between the homogenized elastic tensor obtained with the primal and the dual Hashin-Shtrikman variational principles:

$$
\mathbb{S}_{N}^{\eta}=\left(\mathbb{C}_{N}^{\tau}\right)^{-1}
$$

Consequently, it not possible to obtain new estimate for the homogenized elastic tensor with the dual principle.

Proof: In equation (47) we replace the eigenstrain $\widehat{\boldsymbol{\eta}}_{n}$ by $-\mathbb{S}^{0}: \widehat{\boldsymbol{\rho}}_{n}$. Additionally, the multiplication of relation $(47)$ by $\mathbb{S}^{0}$ leads to:

$$
-\sum_{\alpha=1}^{\alpha=p} \mathbb{S}^{0}:\left(\delta \mathbb{S}^{\alpha}\right)^{-1}: \mathbb{S}^{0}: \widehat{\boldsymbol{\rho}}_{n}^{\alpha}-\mathbb{S}^{0}: \widehat{\boldsymbol{\Delta}}_{n}^{0}: \mathbb{S}^{0}: \widehat{\boldsymbol{\rho}}_{n}=\delta_{n} \mathbb{S}^{0}: \boldsymbol{\Sigma}
$$

In the above equation, we now use the following property:

$$
\mathbb{S}^{0}: \widehat{\boldsymbol{\Delta}}_{n}^{0}: \mathbb{S}^{0}: \widehat{\boldsymbol{\rho}}_{n}=\mathbb{S}^{0}:\left[\widehat{\boldsymbol{\rho}}_{n}-\widehat{\boldsymbol{\rho}}_{0} \delta_{n}\right]-\widehat{\boldsymbol{\Gamma}}_{n}^{0}: \widehat{\boldsymbol{\rho}}_{n}
$$

together with

$$
\mathbb{S}^{0}:\left(\delta \mathbb{S}^{\alpha}\right)^{-1}: \mathbb{S}^{0}=-\mathbb{S}^{0}-\left(\delta \mathbb{C}^{\alpha}\right)^{-1}
$$

It follows that (51) becomes:

$$
\sum_{\alpha=1}^{\alpha=p}\left(\delta \mathbb{C}^{\alpha}\right)^{-1}: \widehat{\boldsymbol{\rho}}_{n}^{\alpha}+\widehat{\boldsymbol{\Gamma}}_{n}^{0}: \widehat{\boldsymbol{\rho}}_{n}=\delta_{n} \mathbb{S}^{0}:\left(\boldsymbol{\Sigma}-\widehat{\boldsymbol{\rho}}_{0}\right)
$$


That is rigorously equivalent to (31) if we replace $\boldsymbol{E}$ by $\mathbb{S}^{0}:\left(\boldsymbol{\Sigma}-\boldsymbol{\rho}_{0}\right)$. Moreover, since $\widehat{\boldsymbol{\tau}}_{0}=\left(\mathbb{C}_{N}^{\tau}-\mathbb{C}^{0}\right): \boldsymbol{E}$, we deduce that:

$$
\widehat{\boldsymbol{\rho}}_{0}=\left(\mathbb{C}_{N}^{\tau}-\mathbb{C}^{0}\right): \mathbb{S}^{0}:\left(\boldsymbol{\Sigma}-\widehat{\boldsymbol{\rho}}_{0}\right)
$$

whose solution is:

$$
\widehat{\boldsymbol{\rho}}_{0}=-\mathbb{C}^{0}:\left[\left(\mathbb{C}_{N}^{\tau}\right)^{-1}-\mathbb{S}^{0}\right]: \Sigma
$$

The solution of (47) for $\widehat{\boldsymbol{\eta}}_{0}$, is then:

$$
\widehat{\boldsymbol{\eta}}_{0}=-\mathbb{S}^{0}: \widehat{\boldsymbol{\rho}}_{0}=\left[\left(\mathbb{C}_{N}^{\tau}\right)^{-1}-\mathbb{S}^{0}\right]: \Sigma
$$

which, by identification with $\widehat{\boldsymbol{\eta}}_{0}=\left(\mathbb{S}_{N}^{\eta}-\mathbb{S}^{0}\right): \boldsymbol{\Sigma}$, proves that $\mathbb{S}_{N}^{\eta}=\left(\mathbb{C}_{N}^{\tau}\right)^{-1}$.

\section{Numerical integration of FFT iterative schemes on the double grid}

The implementation of the iterative schemes involves the discrete convolution product between the Fourier coefficients of the characteristic functions with the components of a second order tensor that is the strain, the stress or the polarization. Since $n$ and $m$ vary from $-N$ to $N-1$, then $n-m$ vary from $-2 N$ to $2 N-2$. It follows that in equation (18), $\widehat{I}_{n-m}^{\alpha}$ must be computed on a double grid while the coefficients $\widehat{y}_{n}$ are computed on the simple grid. This section provides the details about the numerical computation of this discrete convolution product.

Let us introduce

$$
\widehat{y}_{n}^{\times 2}= \begin{cases}0 & \text { if }-2 N \leq n<-N \\ \widehat{y}_{n} & \text { if }-N \leq n \leq N-1 \\ 0 & \text { if } N-1<n \leq 2 N-1\end{cases}
$$

which can be formally written by the linear mapping:

$$
\widehat{Y}^{\times 2}=J \widehat{Y}
$$

where $\widehat{Y}$ and $\widehat{Y}^{\times 2}$ represent the column vector having the components $\widehat{y}_{n}$ and $\widehat{y}_{n}^{\times 2}$ respectively. The vector $\widehat{Y}$ is of dimension $(2 N)^{d}$ while $\widehat{Y}^{\times 2}$ is of dimension 
$(4 N)^{d}$ for $d$-dimensional problems. Moreover, $J$ is a rectangular matrix of dimension $(4 N)^{d} \times(2 N)^{d}$.

The inverse discrete Fourier transform of $\widehat{y}_{n}^{\times 2}$ is:

$$
y_{n}^{\times 2}=\sum_{k=-2 N}^{k=2 N-1} \widehat{y}_{k}^{\times 2} \exp \left(i \underline{\xi}_{k} \cdot \underline{x}_{n}\right)
$$

Here, $\widehat{y}_{n}^{\times 2}$ and $y_{n}^{\times 2}$ are defined on the double grid. The inverse discrete Fourier transform can be formally written as a linear mapping:

$$
Y^{\times 2}=\bar{F} \widehat{Y}^{\times 2}
$$

Similarly, we denote by $I_{n}^{\alpha}$ the inverse Fourier transform of $\widehat{I}_{n}^{\alpha}$, also computed on the double grid

$$
I_{n}^{\alpha}=\sum_{k=-2 N}^{k=2 N-1} \widehat{I}_{n}^{\alpha} \exp \left(i \underline{\xi}_{k} \cdot \underline{x}_{n}\right)
$$

that we denote:

$$
I^{\alpha}=\bar{F} \widehat{I}^{\alpha}
$$

The convolution product (18) is computed by:

$$
\widehat{Y}^{\alpha}=J^{T} F\left(I^{\alpha} \odot Y^{\times 2}\right)
$$

where $J^{T}$ represents the transpose of $J, F$ is the discrete Fourier transform and " $\odot$ " the Hadamard product of two vectors.

Proof: Let us introduce $\widehat{b}=F\left(I^{\alpha} \odot Y^{\times 2}\right)$ whose components are

$$
\widehat{b}_{n}=\frac{1}{(4 N)^{d}} \sum_{k=-2 N}^{2 N-1} I_{k}^{\alpha} y_{k}^{\times 2} \exp \left(-i \underline{\xi}_{n} \cdot \underline{x}_{k}\right)
$$

Introducing (60) and (62) in the above expression leads to:

$$
\begin{aligned}
\widehat{b}_{n} & =\frac{1}{(4 N)^{d}} \sum_{k=-2 N}^{k=2 N-1} \sum_{r=-2 N}^{r=2 N-1} \sum_{m=-2 N}^{m=2 N-1} \widehat{I}_{r}^{\alpha} \widehat{y}_{m}^{\times 2} \exp \left[i\left(\underline{\xi}_{r}+\underline{\xi}_{m}-\underline{\xi}_{n}\right) \cdot \underline{x}_{k}\right] \\
& =\sum_{r=-2 N}^{r=2 N-1} \sum_{m=-2 N}^{m=2 N-1} H_{n r m} \widehat{I}_{r}^{\alpha} \widehat{a}_{m}^{\times 2}
\end{aligned}
$$


in which we have introduced

$$
H_{n r m}=\frac{1}{(4 N)^{d}} \sum_{k=-2 N}^{k=2 N-1} \exp \left[i\left(\underline{\xi}_{r}+\underline{\xi}_{m}-\underline{\xi}_{n}\right) \cdot \underline{x}_{k}\right]
$$

The latter expression is null if $r+m-n \neq 0$, but is equal to 1 if $r+m-n=0$. Eliminating $r$ in (66) leads to

$$
\widehat{b}_{n}=\sum_{m=-2 N}^{m=2 N-1} \widehat{I}_{n-m}^{\alpha} \widehat{y}_{m}^{\times 2}
$$

Moreover, replacing $\widehat{y}_{m}^{\times 2}$ by (59), we obtain:

$$
\widehat{b}_{n}=\sum_{m=-N}^{m=N-1} \widehat{I}_{n-m}^{\alpha} \widehat{y}_{m}
$$

Which proves relation (64) when keeping only the components $\widehat{b}_{n}$ for $-N \leq$ $n \leq N-1$.

The convolution product between the characteristic functions and the components of $\widehat{f}_{n}$ (which represents the components of the strain, the stress or the polarization) is made in four step. First, the components of $\widehat{f}_{n}$ are arranged along the double grid following the linear mapping (59). The inverse discrete Fourier transform of the characteristic functions and $\widehat{f}_{n}$ are computed following (61) and (63), for which the inverse FFT algorithm is used. In the last step, the discrete convolution product is performed following relation (64) for which, again, the FFT is used.

When this convolution has to be made at each step of a recurrence relation, the strain, the stress or the polarization changes from one iteration to another but the characteristic functions remains unchanged during all the iterative process. The components $I_{n}^{\alpha}$ must be computed before the iteration process and stored. The procedure is computationally more expensive than in [28] because the convolution product is made on the double grid while the former method uses a representation of the elasticity tensor on the simple grid. However, the representation on the double grid is necessary to compute exactly the discrete convolution product and then to ensure the bound character of the solutions. Moreover, the method accounts for the real geometry of the cell when exact Fourier coefficients of the characteristic function are used while, in [28], the product between the elastic tensor with the strain is made with the approximation:

$$
\widehat{\varepsilon}_{n}^{\alpha} \sim F F T\left[I^{\alpha}\left(x_{n}\right) F F T^{-1}\left(\varepsilon_{n}\right)\right]
$$


in which $I^{\alpha}\left(x_{n}\right)$ are the values of $I^{\alpha}(\underline{x})$ computed at the nodes of a simple regular grid in the real space.

The shape functions account for the real geometry of the unit cell when the exact expressions of these functions could be derived. For example, for the case of a composite containing fibers with circular cross section, the Fourier transform of the characteristic function can be analytically computed as function of the Bessel function. In this case, there is no approximations on the geometry, while, in the former method [28], the real image was replaced by a pixelized image which approximates the inclusion geometry by means of rectangular elements. Note also that finite element methods also approximate the real geometry since they use triangular elements (or other kinds of elements) in order to mesh the inclusion. Truly curved objects are then approximated by polygon meshes.

However, the exact Fourier transform of these shape functions can be obtained analytically only for some particular cases, including elliptic or rectangular inclusions. This expressions have been used in Nemat-Nasser and Hori [32] to obtain estimates of the effective properties of composites materials and have been also considered by Bonnet [4] in order to improve the solutions computed from FFT based iterative schemes. The analytic expressions for rectangular inclusions can be also considered when dealing with digital images obtained from microtomography. For other inclusion shapes, such as the "flower" inclusion considered in section 6.2, there is no analytic expressions of the shape functions and the Fourier transform must be computed by using a mesh of the phases. In this case, the exact geometry is then not accounted by the method and the approximations introduced by the discretization are comparable to that made with finite element.

\section{Hierarchization of FFT solutions}

Different FFT solutions are derived from variational principles: two solutions for the strain and the stress coming from the classic principles and an infinite number of solutions for the polarization which depend on the choice of the reference material. We aim now at comparing these solutions. In section 6.1, we establish inequalities between the classic bounds and the Hashin-Shtrikman one (obtained in the range $\mathbb{C}^{0}>0$ ). Moreover, the polarization scheme is generally considered with $\mathbb{C}^{0}<0$ in the literature $[26,31]$ since in this range, the iterative process has a good rate of convergence. Consequently a comparison with classic bounds for $\mathbb{C}^{0}<0$ is also investigated in section 6.2 . 


\subsection{Case $\mathbb{C}^{0}>0$}

For any polarization tensor $\boldsymbol{\tau}$ and for any kinematically admissible strain field $\varepsilon$, we have:

$$
\begin{aligned}
\mathcal{U}(\varepsilon)-\mathcal{H}(\boldsymbol{\tau})= & \frac{1}{2}<\left(\boldsymbol{\tau}+\mathbb{C}^{0}: \boldsymbol{\varepsilon}\right): \Gamma^{0} *\left(\boldsymbol{\tau}+\mathbb{C}^{0}: \boldsymbol{\varepsilon}\right)>_{V} \\
& +\frac{1}{2}<(\boldsymbol{\tau}-\delta \mathbb{C}: \varepsilon): \delta \mathbb{C}^{-1}:(\boldsymbol{\tau}-\delta \mathbb{C}: \varepsilon)>_{V}
\end{aligned}
$$

Proof: by expanding each terms on the right side of the equality in (71) we obtain:

$$
\begin{aligned}
\mathcal{U}(\varepsilon)-\mathcal{H}(\boldsymbol{\tau})= & \frac{1}{2}<\boldsymbol{\tau}: \Gamma^{0} * \boldsymbol{\tau}>_{V}+<\boldsymbol{\tau}: \Gamma^{0} *\left(\mathbb{C}^{0}: \varepsilon\right)>_{V} \\
& +\frac{1}{2}<\varepsilon: \mathbb{C}^{0}: \Gamma^{0} *\left(\mathbb{C}^{0}: \varepsilon\right)>_{V}+\frac{1}{2}<\boldsymbol{\tau}: \delta \mathbb{C}^{-1}: \boldsymbol{\tau}>_{\mathbb{V}}(72) \\
& -<\boldsymbol{\tau}: \boldsymbol{\varepsilon}>_{V}+\frac{1}{2}<\boldsymbol{\varepsilon}: \delta \mathbb{C}: \varepsilon>_{V}
\end{aligned}
$$

Moreover, for any admissible strain field $\varepsilon$, we have:

$$
\Gamma^{0} *\left(\mathbb{C}^{0}: \varepsilon\right)=\varepsilon-\boldsymbol{E}
$$

It follows that (72) becomes:

$$
\begin{aligned}
\mathcal{U}(\varepsilon)-\mathcal{H}(\boldsymbol{\tau})= & \frac{1}{2}<\boldsymbol{\tau}: \Gamma^{0} * \boldsymbol{\tau}>_{V}-<\boldsymbol{\tau}>_{V}: \boldsymbol{E} \\
& -\frac{1}{2} \boldsymbol{E}: \mathbb{C}^{0}: \boldsymbol{E}+\frac{1}{2}<\boldsymbol{\tau}: \delta \mathbb{C}^{-1}: \boldsymbol{\tau}>_{V} \\
& +\frac{1}{2}<\varepsilon: \mathbb{C}: \boldsymbol{\varepsilon}>_{V}
\end{aligned}
$$

It can be observed that with the definition (12) for $\mathcal{U}(\varepsilon)$ and $(27)$ for $\mathcal{H}(\boldsymbol{\tau})$, the expressions at each sides of (74) are rigorously equivalent, which proves relation (71).

Now, if $\mathbb{C}^{0}>\mathbb{C}$, then $\delta \mathbb{C}$ is negative definite and the last term in (71) is surely negative whatever the value of $\boldsymbol{\tau}$ and $\boldsymbol{\varepsilon}$. In this case, we have the inequality:

$$
\mathcal{U}(\varepsilon)-\mathcal{H}(\boldsymbol{\tau}) \leq \frac{1}{2}<\left(\boldsymbol{\tau}+\mathbb{C}^{0}: \varepsilon\right): \Gamma^{0} *\left(\boldsymbol{\tau}+\mathbb{C}^{0}: \varepsilon\right)>_{V}
$$


Now we use (75) to establish an inequality for the solution obtained with the classic variational principle based on the strain energy $\mathcal{U}(\varepsilon)$ and the solution derived from the Hashin-Shtrikman variational principle. Considering a strain $\varepsilon \in \mathcal{E}_{N}$ and a polarization $\tau \in \mathcal{T}_{N}$, inequality (75) becomes (and making use of the Parseval theorem):

$$
\widetilde{\mathcal{U}}\left(\widehat{\boldsymbol{\varepsilon}}_{n}\right)-\widetilde{\mathcal{H}}\left(\widehat{\boldsymbol{\tau}}_{n}\right) \leq \frac{1}{2} \sum_{n=-N}^{n=N-1}\left(\overline{\widehat{\boldsymbol{\tau}}_{n}}+\mathbb{C}^{0}: \overline{\widehat{\boldsymbol{\varepsilon}}_{n}}\right): \widehat{\boldsymbol{\Gamma}}_{n}^{0}:\left(\widehat{\boldsymbol{\tau}}_{n}+\mathbb{C}^{0}: \widehat{\boldsymbol{\varepsilon}}_{n}\right)
$$

where $\widetilde{\mathcal{U}}\left(\widehat{\boldsymbol{\varepsilon}}_{n}\right)$ and $\widetilde{\mathcal{H}}\left(\widehat{\boldsymbol{\tau}}_{n}\right)$ are given by (19) and (30) respectively. To avoid any confusion, let us denote by $\widehat{\boldsymbol{\varepsilon}}_{n}^{*}$ and $\widehat{\boldsymbol{\tau}}_{n}^{*}$ the stationary point of $\widetilde{\mathcal{U}}\left(\widehat{\boldsymbol{\varepsilon}}_{n}\right)$ and $\widetilde{\mathcal{H}}\left(\widehat{\boldsymbol{\tau}}_{n}\right)$ which are solutions of the linear systems (20) and (31) respectively. The classic variational principle implies that for any $\varepsilon \in \mathcal{E}_{N}$ we have:

$$
\tilde{\mathcal{U}}\left(\widehat{\varepsilon}_{n}^{*}\right) \leq \tilde{\mathcal{U}}\left(\widehat{\varepsilon}_{n}\right)
$$

Moreover, consider in (76) the kinematically admissible strain:

$$
\widehat{\varepsilon}_{n}=\delta_{n} \boldsymbol{E}-\widehat{\boldsymbol{\Gamma}}_{n}^{0}: \widehat{\boldsymbol{\tau}}_{n}^{*}
$$

The term at the right of the inequality in (76) is null because:

$$
\widehat{\boldsymbol{\Gamma}}_{n}^{0}: \mathbb{C}^{0}:\left(\delta_{n} \boldsymbol{E}-\widehat{\boldsymbol{\Gamma}}_{n}^{0}: \widehat{\boldsymbol{\tau}}_{n}^{*}\right)=-\widehat{\boldsymbol{\Gamma}}_{n}^{0}: \widehat{\boldsymbol{\tau}}_{n}^{*}
$$

Accounting for (77) it follows that:

$$
\widetilde{\mathcal{U}}\left(\widehat{\varepsilon}_{n}^{*}\right) \leq \widetilde{\mathcal{H}}\left(\widehat{\tau}_{n}^{*}\right)
$$

and then:

$$
\mathbb{C}_{N}^{\varepsilon} \leq \underset{\mathbb{C}^{0}>\mathbb{C}}{\mathbb{C}_{N}^{\tau}\left(\mathbb{C}^{0}\right)}
$$

Consequently, it is not possible to derive a better upper bound with the Hashin-Shtrikman variational principle than with the classic principle when the same discretization is used for both the strain and the polarization.

By duality, for any eigenstrain $\boldsymbol{\eta}$ and for any statically admissible stress field $\boldsymbol{\sigma}$, we have: 


$$
\begin{aligned}
\mathcal{W}(\boldsymbol{\sigma})-\mathcal{L}(\boldsymbol{\eta})= & \frac{1}{2}<\left(\boldsymbol{\eta}+\mathbb{S}^{0}: \boldsymbol{\sigma}\right): \Delta^{0} *\left(\boldsymbol{\eta}+\mathbb{S}^{0}: \boldsymbol{\sigma}\right)>_{V} \\
& +\frac{1}{2}<(\boldsymbol{\eta}-\delta \mathbb{S}: \boldsymbol{\sigma}): \delta \mathbb{S}^{-1}:(\boldsymbol{\eta}-\delta \mathbb{S}: \boldsymbol{\sigma})>_{V}
\end{aligned}
$$

that is proved with a similar demonstration that used for (71). If $0<\mathbb{C}^{0}<\mathbb{C}$, then $\mathbb{S}^{0}>\mathbb{S}$ and $\delta \mathbb{S}$ is negative definite. The following inequality then holds for any eigenstrain $\boldsymbol{\eta}$ and for any statically admissible stress field $\boldsymbol{\sigma}$ :

$$
\mathcal{W}(\varepsilon)-\mathcal{L}(\boldsymbol{\eta}) \leq \frac{1}{2}<\left(\boldsymbol{\eta}+\mathbb{S}^{0}: \boldsymbol{\sigma}\right): \Delta^{0} *\left(\boldsymbol{\eta}+\mathbb{S}^{0}: \boldsymbol{\sigma}\right)>_{V}
$$

Following the same demonstration, we conclude that:

$$
\widetilde{\mathcal{W}}\left(\widehat{\boldsymbol{\sigma}}_{n}^{*}\right) \leq \widetilde{\mathcal{L}}\left(\widehat{\boldsymbol{\eta}}_{n}^{*}\right)
$$

and then:

$$
\mathbb{S}_{N}^{\sigma} \leq \mathbb{S}_{N}^{\eta}\left(\mathbb{C}^{0}\right)
$$

Moreover, since $\left(\mathbb{S}_{N}^{\eta}\right)^{-1}=\left(\mathbb{C}_{N}^{\tau}\right)^{-1}$ we have also:

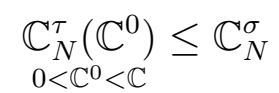

and accounting for (14) and (81), we deduce that:

$$
\underset{0<\mathbb{C}^{0}<\mathbb{C}}{\mathbb{C}_{N}^{\tau}\left(\mathbb{C}^{0}\right)} \leq \mathbb{C}_{N}^{\sigma} \leq \mathbb{C}^{h o m} \leq \mathbb{C}_{N}^{\varepsilon} \leq \underset{\mathbb{C}^{0}>\mathbb{C}}{\mathbb{C}_{N}^{\tau}\left(\mathbb{C}^{0}\right)}
$$

As an illustration purpose, we consider the $2 \mathrm{~d}$ problem depicted in figure 1 . The unit cell contains a circular inclusion and is subjected to the macroscopic strain $E_{11}=1, E_{22}=-1$ and $E_{12}=0$ or the macroscopic stress $\Sigma_{11}=1$, $\Sigma_{22}=-1$ and $\Sigma_{12}=0$. We assume that both the matrix and the inclusion are incompressible and we denote $\mu_{1}$ and $\mu_{2}$ their elastic shear modulus. The problem is solved with 128 wave vectors along each space directions and we put $\mu_{1}=1$ and $\mu_{2}=10$. 


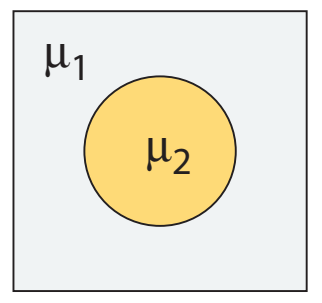

Fig. 1. Unit cell containing a circular inclusion with shear moduli $\mu_{1}$ and $\mu_{2}$.

in figure 2 we represent the macroscopic shear elastic modulus, computed with the polarization scheme, as function of the shear modulus of the reference material in the range $\mu_{0}>0$. Are also reported on this figure the upper and the lower bound based on the strain and the stress formulation (the two horizontal lines). In the range $\mu_{0}>10$, the Hashin-Shtrikman principle provides an upper bound that is less accurate that the strain based one. Alternatively, in the range $0<\mu_{0}<1$, the stress based bound is always better than one derived by the Hashin-Shtrikman approach. These numerical results are conform with relations (87). Moreover, it is also observed that in the limit $\mu_{0} \rightarrow+\infty$ (resp. $\mu_{0} \rightarrow 0$ ), the two lower bounds (resp. the two upper bounds) coincide, as suggested by the two relations in (45).

Effective shear modulus

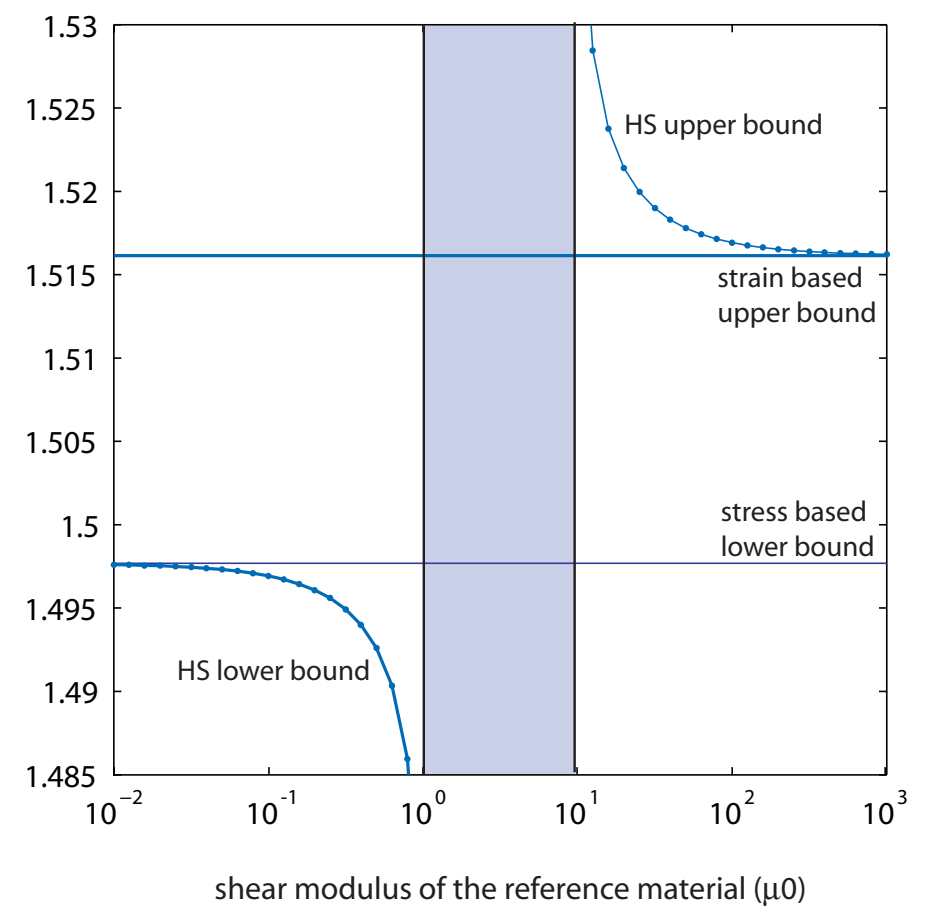

Fig. 2. Effective shear modulus computed with the Hashin-Shtrikman approach as function of $\mu_{0}$ in the range $\mu_{0}>0$. The two horizontal lines correspond to the strain and stress based bounds.

For completeness, we represent in figure (3) the number of iterations at con- 
vergence of the polarization-based iterative scheme as function of the shear modulus $\mu_{0}$ in the range $\mu_{0}>10$ and in the range $0<\mu_{0}<1$. Are also reported on these figures (the two horizontal lines) the number of iteration at convergence of the strain and stress based iterative schemes, obtained with the optimal shear moduli:

$$
\mu_{0(\text { strain })}=\frac{1}{2}\left(\mu_{1}+\mu_{2}\right), \quad \mu_{0(\text { stress })}=\left[\frac{1}{2}\left(\frac{1}{\mu_{1}}+\frac{1}{\mu_{2}}\right)\right]^{-1}
$$

The value $\mu_{0 \text { (strain) }}$ has been proved, in [22], to minimize the spectral radius of the linear operator involved in the strain based recurrence relation. By making a similar analysis, it can be shown that $\mu_{0 \text { (stress) }}$ is optimal for the stress based iterative scheme.

Clearly, the results show that the rate of convergence of the strain and the stress based iterative scheme is better than the rate of convergence of the polarization based iterative scheme. Since the polarization based iterative scheme does not provides better bounds and does not improve the rate of convergence, it has no real computational interest when it is used with $\mathbb{C}^{0}>0$.

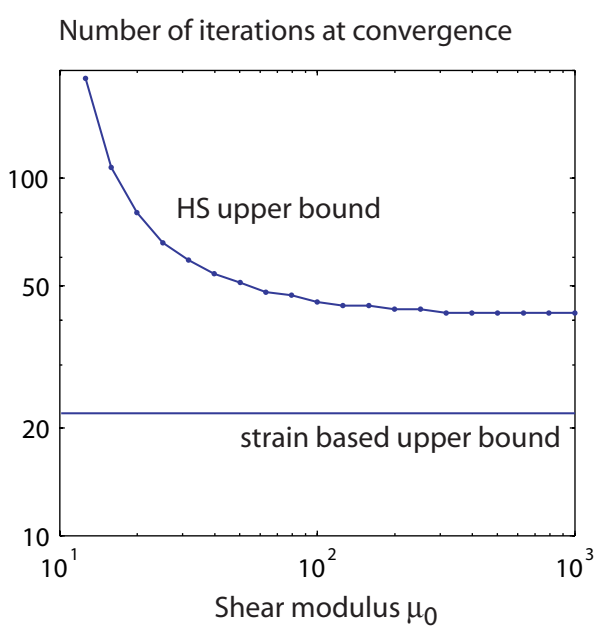

Number of iterations at convergence

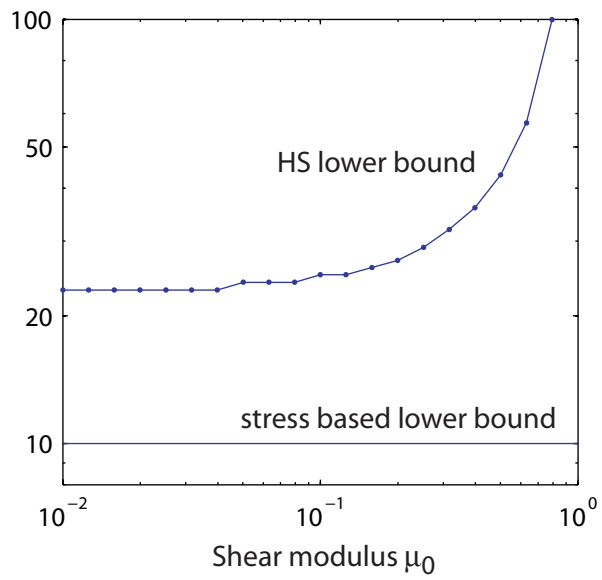

Fig. 3. Number of iterations at convergence of the polarization based iterative scheme as function of $\mu_{0}$, in the range $0<\mu_{0}<1$ (at the left) and $\mu_{0}>1$ (at the right). The two horizontal lines correspond to the number of iterations needed to obtain the convergence of the strain and the stress based iterative schemes.

\subsection{Case $\mathbb{C}^{0}<0$}

We now consider the polarization based iterative scheme when $\mathbb{C}^{0}<0$. As shown in [26], the use of the polarization with $\mathbb{C}^{0}<0$ is interesting since it drastically improves the rate of convergence of the strain and stress based ones, and particularly in the domain of high contrasts. However, when $\mathbb{C}^{0}<0$ 
the Hashin-Shtrikman variational principle does not deliver a bound for the effective properties and the solutions must be considered only as an estimate for the effective elasticity coefficients.

In order to evaluate the accuracy of solution for $\mathbb{C}^{0}<0$, let us consider again the problem of the circular unit cell represented in figure 1. In figure 4 the effective shear modulus $\mu^{\text {hom }}$ computed with the polarization based iterative scheme is represented as function of the shear modulus of the reference material in the range $\mu_{0}<0$. On this figure, the lower and the upper bound computed with the strain and the stress based iterative schemes are also provided. It can be observed that the solution obtained with the polarization is comprised between the two classic bounds (strain and stress based). Moreover, and as expected by relations (45), the polarization based estimate coincides with the upper bound when $\mu_{0} \rightarrow-\infty$ and coincides with the lower bound when $\mu_{0} \rightarrow 0$. This observation suggests to call this solution as "intermediate" (in fact intermediate between the classic lower and upper bounds).

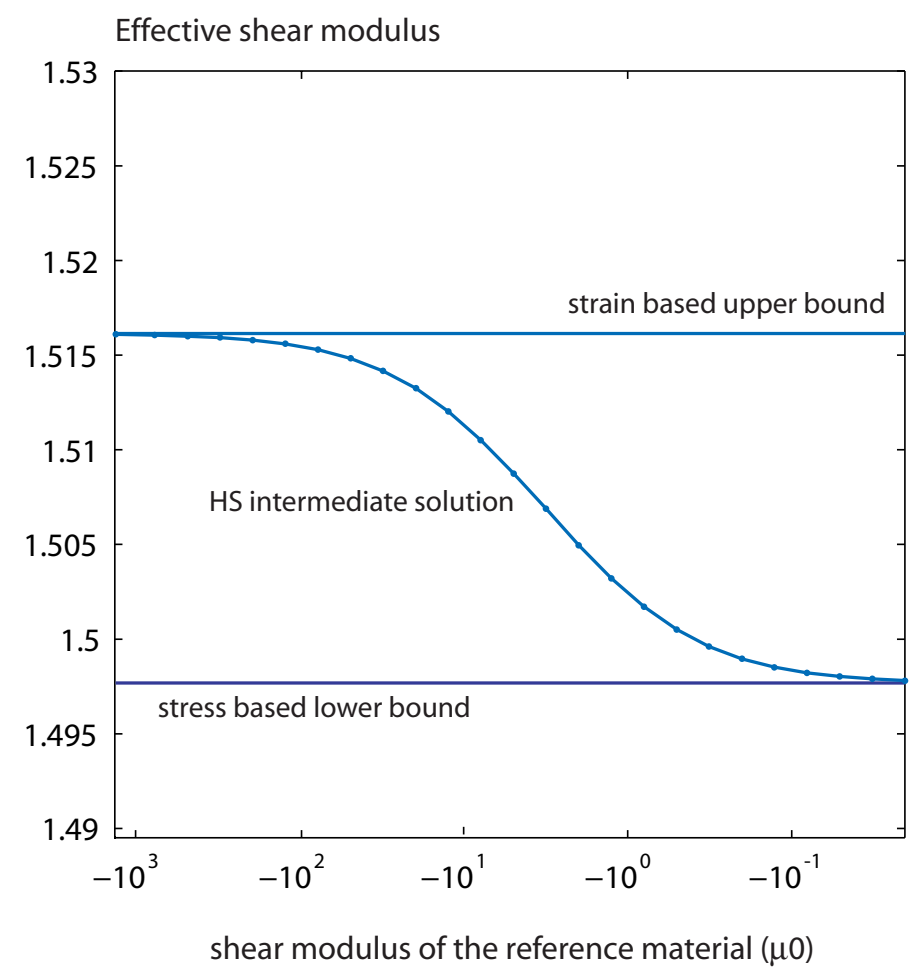

Fig. 4. Effective shear modulus computed with the Hashin-Shtrikman approach as function of $\mu_{0}$ in the range $\mu_{0}<0$

Let us introduce the quantity:

$$
Y\left(\mu_{0}\right)=\frac{\mu_{N}^{\tau}\left(\mu_{0}\right)-\mu_{N}^{\sigma}}{\mu_{N}^{\varepsilon}-\mu_{N}^{\sigma}}
$$


where $\mu_{N}^{\varepsilon}, \mu_{N}^{\sigma}$ and $\mu_{N}^{\tau}\left(\mu_{0}\right)$ are the effective shear modulus computed with the strain, the stress and the polarization based iterative scheme respectively. When $\mu_{N}^{\tau}\left(\mu_{0}\right)$ is comprised between the upper and the lower bounds (resp. $\mu_{N}^{\varepsilon}$ and $\left.\mu_{N}^{\sigma}\right)$, the quantity $Y\left(\mu_{0}\right)$ is then comprised between 0 and 1 . We propose to evaluate the value of this quantity with numerical examples with different contrasts and shapes of inclusion.

In figure (5), we now provide the variations of the quantity $Y\left(\mu_{0}\right)$ with $\mu_{0}<0$ for three values of the contrast: $\mu_{I} / \mu_{M}=100, \mu_{I} / \mu_{M}=10, \mu_{I} / \mu_{M}=0.1$. In figure (6), we represent the same quantity $Y\left(\mu_{0}\right)$ for two other shape of inclusion: the square and the flower inclusion and for the contrast $\mu_{I} / \mu_{M}=10$. It can be observed that in each cases, $Y\left(\mu_{0}\right)$ is comprised between 0 and 1 which means that the polarization scheme provide a solution for the effective shear modulus that is always comprised between the two classic bounds.

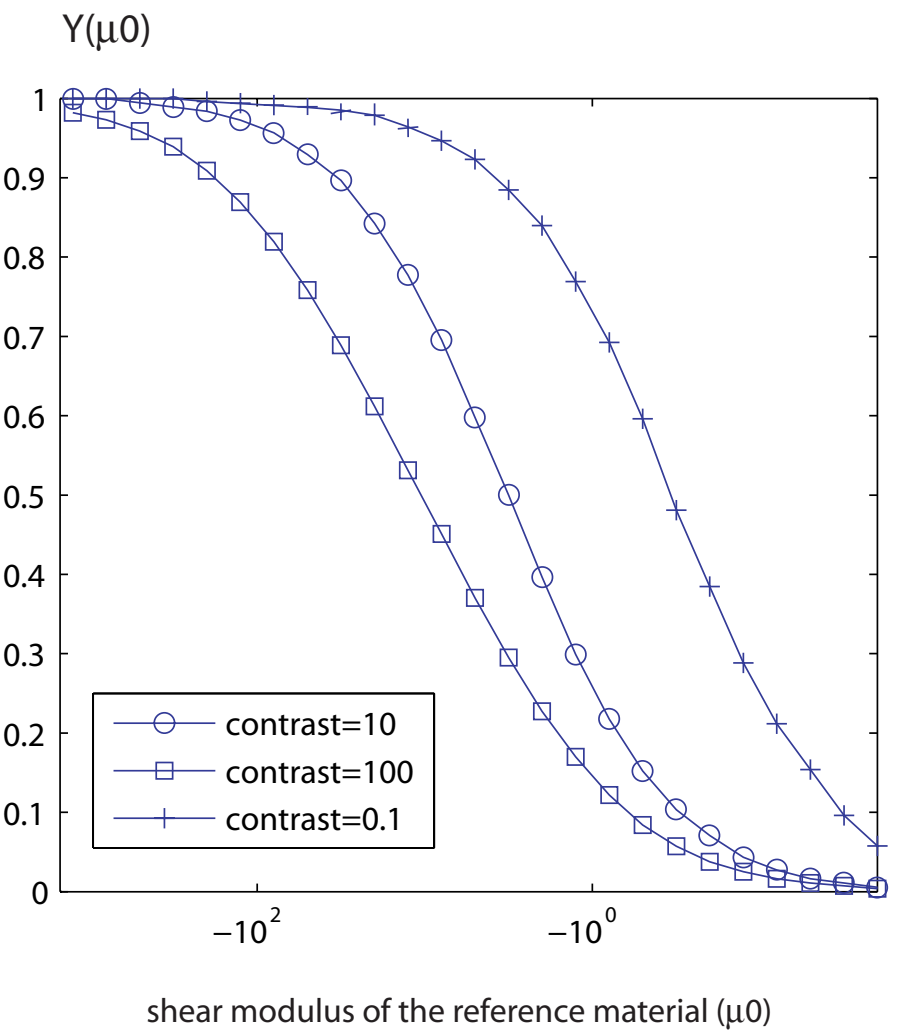

Fig. 5. Variations of quantity $Y\left(\mu_{0}\right)$ as function of $\mu_{0}$ for the circular inclusion with three values of the contrast. 

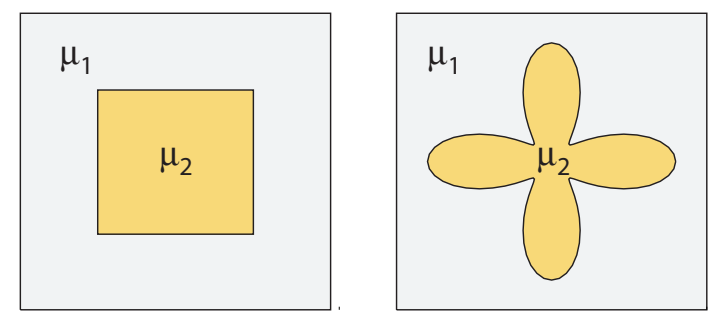

Fig. 6. Unit cells containing a rectangular or a flower inclusion.

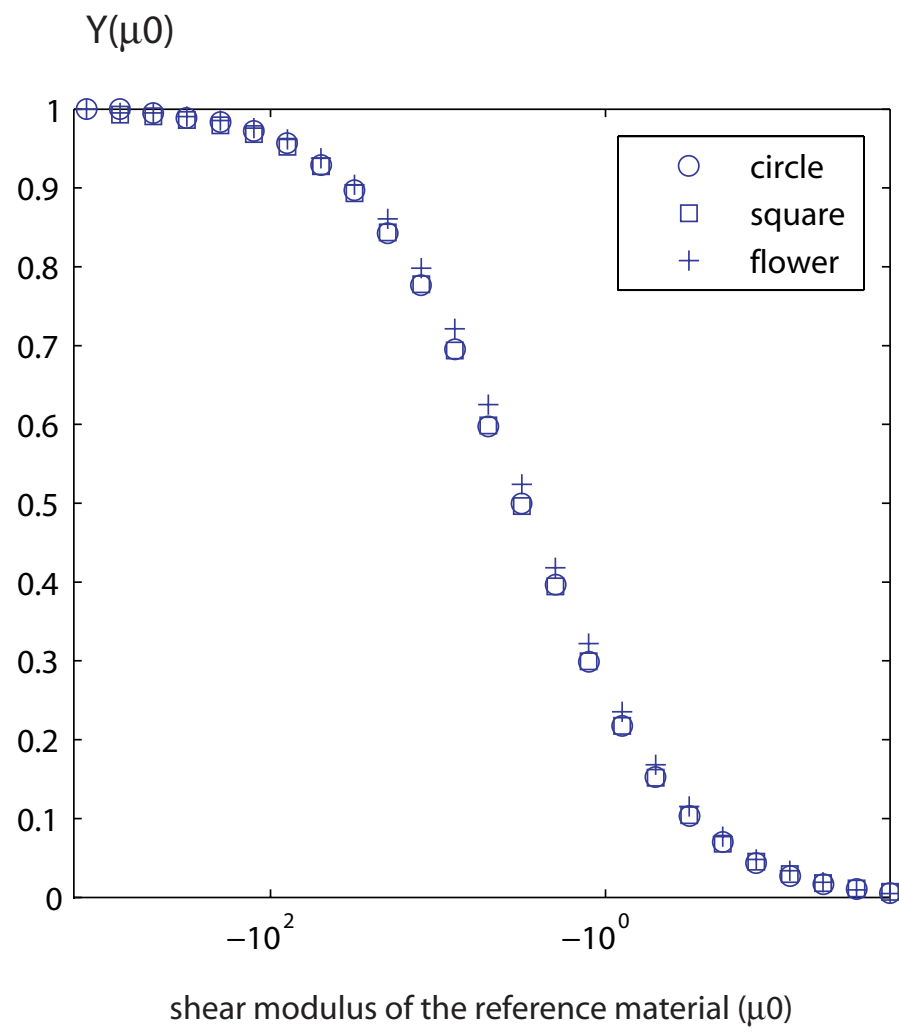

Fig. 7. Variations of quantity $Y\left(\mu_{0}\right)$ as function of $\mu_{0}$ for the circular, the rectangular and the flower inclusion and for the contrast of 10 .

These numerical results suggest that:

$$
\underset{0<\mathbb{C}^{0}<\mathbb{C}}{\mathbb{C}_{N}^{\tau}\left(\mathbb{C}^{0}\right)} \leq \mathbb{C}_{N}^{\sigma} \leq \underset{\mathbb{C}^{0}<0}{\mathbb{C}_{N}^{\tau}\left(\mathbb{C}^{0}\right)} \leq \mathbb{C}_{N}^{\varepsilon} \leq \underset{\mathbb{C}^{0}>\mathbb{C}}{\mathbb{C}_{N}^{\tau}\left(\mathbb{C}^{0}\right)}
$$

However this inequalities cannot been established rigorously since in the range $\mathbb{C}<0, \mathcal{H}(\boldsymbol{\tau})$ is not convex, nor concave. Moreover, and for the same reasons, it is also not possible to establish an inequality with $\mathbb{C}^{\text {hom }}$. In the range $\mathbb{C}^{0}<0$ the accuracy of this solution is then uncertain. 


\section{Conclusion}

In this paper, we have proposed a unified variational approach of FFT based iterative schemes. Classic variational approaches, based on the strain and stress elastic potentials, are first considered for the derivation of an upper and a lower bounds for the effective properties of the composite. The stationary point is determined by means of fixed-point iterative schemes which use the Green operator. The Hashin-Shtrikman principle is also considered and the associated linear system is solved by means of a more sophisticated algorithm based on the polarization. Depending on the choice of the elastic modulus of the reference material (positive or negative), the solution for the polarization leads to either an upper bound, a lower bound or an estimate for the homogenized elastic tensor. The main feature of these iterative schemes lies in the representation of the elasticity tensor on the double grid which advantageously account for the exact geometry of the cell. Consequently, the methods deliver rigorous bounds for the effective elasticity tensor. A hierarchization of the different FFT solutions is afterward investigated. It has been shown that the numerical Hashin-Shtrikman bounds are less accurate that the classic bounds (based on the strain and stress). Moreover, when positive values for the elastic moduli of the reference material are considered, it has been observed that the rate of convergence of the polarization based iterative scheme is also reduced comparatively to the rate of convergence of the strain and stress based iterative schemes. We conclude that the Hashin-Shtrikman variational principle and the associated polarization based iterative scheme are irrelevant when used with positive values of the elastic tensor of the reference material. Considering now the polarization based iterative scheme with negative values of the elastic tensor of the reference material, for which the rate of convergence polarization scheme is significantly improved compared to the strain and stress based iterative schemes, the solution is neither a lower or an upper bound but is only on estimate which is strictly comprised between the two classic bounds.

\section{References}

[1] Bhattacharya K., Suquet P.M.

A model problem concerning recoverable strains of shape-memory polycrystals. Proc. Roy. Soc. London A. 2005, vol. 461, pp. 2797-2816.

[2] Bilger N., Auslender F., Bornert M., Michel J.-C., Moulinec H., Suquet P., Zaoui A.

Effect of a nonuniform distribution of voids on the plastic response of voided materials: a computational and statistical analysis. Int. J. Solids Struct. 2005, vol. 42 , pp. 517-538. 
[3] Bilger N., Auslender F., Bornert M., Moulinec H., Zaoui A.

Bounds and estimates for the effective yield surface of porous media with a uniform or a nonuniform distribution of voids. Eur. J. Mech., A/Solids. 2007, vol. 26(5), pp. 810-836.

[4] Bonnet G.

Effective properties of elastic periodic composite media with fibers. J. Mech. Phys. Solids. 2007, vol. 55, pp. 881-899.

[5] Brenner R.

Numerical computation of the response of piezoelectric composites using Fourier transform. Phys. Review B. 2007, vol. 79, 184106.

[6] Brenner R.

Computational approach for composite materials with coupled constitutive laws. Zeitschrift für angewandte Mathematik und Physik. 2012, vol. 61(5) pp. 919-927.

[7] Brisard S. and Dormieux L.

FFT-based methods for the mechanics of composites: A general variational framework. Comput. Mater. Science. 2010, vol. 49(3), pp. 663-671.

[8] Brisard S. and Dormieux L.

Combining Galerkin approximation techniques with the principle of Hashin and Shtrikman to derive a new FFT-based numerical method for the homogenization of composites. Comput. Methods Appl. Mech. Engrg. 2012, vol. 217-220, pp. 197212.

[9] Brown W.

Solid mixture permittivities. J. Comput. Math. 1955, vol. 23, pp. 1514-1517.

[10] Escoda J., Willot F., Jeulina D., Sanahujab J., Toulemondeb C.

Estimation of local stresses and elastic properties of a mortar sample by FFT computation of fields on a 3D image. Cement and Concrete Research. 2011, vol. $41(5), 542-556$.

[11] Eyre D.J. and Milton G.W.

A fast numerical scheme for computing the response of composites using grid refinement. J. Phys. III. 1999, vol. 6, pp. 41-47.

[12] Gélébart L., Mondon-Cancel R.

Non-linear extension of FFT-based methods accelerated by conjugate gradients to evaluate the mechanical behavior of composite materials. Comp. Mater. Science, 2013, vol. 77, pp. 430-439

[13] Hashin Z., Shtrikman S. On some variational principles in anisotropic and nonhomogeneous elasticity. J. Mech. Phys. Sol. 1962, vol. 10 (4), pp. 335-342.

[14] Idiart M.I., Willot F., Pellegrini Y.-P., Ponte Castañeda P.

Infinite-contrast periodic composites with strongly nonlinear behavior: Effective-medium theory versus full-field simulations. Int. J. Solids Structures. 2009, vol. 46, pp. 3365-3382. 
[15] Kroner E.

Statistical Continuum Mechanics (Springer-Verlag, Wien, 1972).

[16] Lebensohn R.A. , Brenner R., Castelnau O., Rollett A.D.

Orientation image-based micromechanical modelling of subgrain texture evolution in polycrystalline copper. Acta Materialia. 2008, vol. 56, pp. 39143926 .

[17] Lebensohn R.A., Kanjarla A.K, Eisenlohr P.

An elasto-viscoplastic formulation based on fast Fourier transforms for the prediction of micromechanical fields in polycrystalline materials. Int. J. Plasticity. 2012, vol. 32-33, pp. 59-69.

[18] Li J., Tian X.-X., Abdelmoula, R. A damage model for crack prediction in brittle and quasi-brittle materials solved by the FFT method. Int. J. Fracture. 2012, vol. 173(2), pp. 135-146.

[19] Li J., Meng S., Tian X., Song F., Jiang C. A non-local fracture model for composite laminates and numerical simulations by using the FFT method. Composites Part B: Engineering. 2012, vol. 43(3), 961-971.

[20] Michel J. C., Moulinec H., Suquet P.

Effective properties of composite materials with periodic microstructure: a computational approach. Comput. Methods. Appl. Mech. Engrg. 1999, vol. 172, pp. 109-143.

[21] Michel J. C., Moulinec H., Suquet P.

A computational method based on augmented lagrangians and fast fourier transforms for composites with high contrast. Comput. Model. Eng. Sci. 2000, vol. 1 (2), pp. 9-88.

[22] Michel J. C., Moulinec H., Suquet P.

A computational scheme for linear and non-linear composites with arbitrary phase contrast. Int. J. Numer. Meth. Engng. 2001, vol. 52, pp. 139-160.

[23] Milton G. W., Kohn R.V.

Variational bounds of the effective of anisotropic composites. Journal of the Mechanics and Physics of Solids, vol 36(6), 597-629, 1988.

[24] Milton G. W.

The Theory of Composites. Cambridge University Press, 2002.

[25] Monchiet V., Bonnet G., Lauriat G. A FFT-based method to compute the permeability induced by a Stokes slip flow through a porous medium. Comptes Rendus Mécanique. Vol. 337(4), pp. 192-197, 2009.

[26] Monchiet V., Bonnet G. A polarization based FFT iterative scheme for computing the effective properties of elastic composites with arbitrary contrast. International Journal for Numerical Methods in Engineering. Vol. 89(11), pp. 1419-1436, 2012. 
[27] Monchiet V., Bonnet G. Numerical homogenization of non linear composites with a polarization-based FFT iterative scheme. Comput. Mater. Science. Vol. 79, pp. 276-283, 2013.

[28] Moulinec H. and Suquet P.

A fast numerical method for computing the linear and nonlinear mechanical properties of composites. C. R. Acad. Sci. 1994, vol. 318 (11), pp. 1417-1423.

[29] Moulinec H. and Suquet P.

A numerical method for computing the overall response of nonlinear composites with complex microstructure. Comput. Methods. Appl. Mech. Engrg. 1998, vol. 157, pp. 69-94.

[30] Moulinec H., Suquet P.

Intraphase strain heterogeneity in nonlinear composites: a computational approach. Eur. J. Mech. A. 2003, vol. 22, pp. 751-770.

[31] Moulinec H., Silva F.

Comparison of three accelerated FFT-based schemes for computing the mechanical response of composite materials. International Journal for Numerical Methods in Engineering. 2014, vol. 97(13), pp. 960-985

[32] Nemat-Nasser S., Hori M.

Micromechanics: Overall Properties of Heterogeneous Materials. NorthHolland, Amsterdam, 1999.

[33] Nguyen T.-K., Monchiet V., Bonnet G. A Fourier based numerical method for computing the dynamic permeability of porous media. European Journal of Mechanics B/fluids. Vol. 37, pp. 90-98, 2013.

[34] P. Ponte Castaneda and P. Suquet, Adv. Appl. Mech. Vol. 34, pp. 171, 1998.

[35] Vinogradov V., Milton G.W.

An accelerated FFT algorithm for thermoelastic and non-linear composites. International Journal for Numerical Methods in Engineering. 2008, vol. 76 (11), pp. 1678-1695.

[36] Vondrejc J.

FFT-based method for homogenization of periodic media: Theory and applications. PhD, Department of Mechanics, Faculty of Civil Engineering, Czech Technical University, Czech Republic, Prague, 2013.

[37] Vondrejc J., Zeman J., Marek I.

An FFT-based Galerkin Method for Homogenization of Periodic Media. 2013, arXiv:1404.3614.

[38] Walpole L.J.

Elastic behavior of composite materials : theoretical foundations. Advances in App. Mech., 21 : 169-243, 1981.

[39] Willis J.R.

Bounds and self-consistent estimates for the overall properties of anisotropic composites. J. Mech. Phys. Solids. 1977, vol. 25 (3), pp. 185-202. 
[40] Willot F., Pellegrini Y.P., Ponte-Castaneda P.

Localization of elastic deformation in strongly anisotropic, porous, linear materials with periodic microstructures: Exact solutions and dilute expansions. J. Mech. Phys. Solids. 2008, vol. 56(4), pp. 1245-1268.

[41] Willot F., Abdallah B., Pellegrini Y.P.

Fourier-based schemes with modified Green operator for computing the electrical response of heterogeneous media with accurate local fields. 2013, arXiv e-print 1307.1015.

[42] Yvonnet J.

A fast method for solving microstructural problems defined by digital images: a space LippmannÜSchwinger scheme. Int. J. Num. Methods Engrg. 2012, vol. 92(2), pp. 178-205.

[43] Zeman J., Vondrejc J., Novák J., Marek I.

Accelerating a FFT-based solver for numerical homogenization of periodic media by conjugate gradients. J. Comput. Physics. 2010, vol. 229(21), pp. 80658071.

[44] Zhykov V.V.

Estimates for the homogenized matrix and the homogenized tensor. Russian Mathematical Survey. 1991, vol. 46, pp. 49-109. 